\title{
Kinetic modelling of compartmentalised reaction networks
}

\author{
Jan-Hendrik S. Hofmeyr \\ Dept. of Biochemistry, University of Stellenbosch, \\ Private Bag X1, Matieland 7602, South Africa
}

April 3, 2020

\section{Contents}

1. Introduction 2

2. Defining reaction and transfer rates 4

3. Clearance 6

4. Transfer rates between compartments $\quad 7$

5. Compartmental geometry 9

6. Interlude: A thought experiment 10

7. Reactions at the interface between compartments 11

8. Transfer through pores or by mediator facilitation 14

9. A unified view of rate equations for multi-compartment kinetic modelling 16

10. Rates of processes at compartment boundaries are never directly propor$\begin{array}{ll}\text { tional to a volume } & 17\end{array}$

11. Incorporating rate equations in ODEs 21

11.1. ODEs for fixed-size single-compartment systems . . . . . . . . . . . 23

11.2. ODEs for fixed-size multi-compartment systems . . . . . . . . . . 25

11.3. ODEs that use amount-change rate equations with rate factors . . . . 27

11.4. ODEs for variable-size compartmentalised systems . . . . . . . . . . 27

12. Moiety conservation 29

13. Advice to modellers and software authors 30

A. The kinetic model 34

B. Example: A compartmentalised Lotka-Volterra system 36

C. PySCeS model definition for the compartmentalised Lotka-Volterra system 37

D. Example: Minimal whole-cell model (MWC-model) 39

E. PySCeS model definition for the minimal whole-cell model 42 


\section{Abstract}

This paper presents a comprehensive treatment of kinetic modelling of compartmentalised reaction networks in the context of systems biology. There is still a lot of confusion about how to go about constructing compartment models, and many published models are flawed with respect to how they handle compartmentation. The modelling framework described here answers two key questions: Which rate laws should be used to describe the rates of reactions in compartmentalised systems? How should these rate laws be incorporated in the ordinary differential equations (ODEs) that describe the dynamics of the compartmentalised system? The framework rests on the fundamental definition of reaction rate as the number of reaction events per time, which is related to the time derivative of mole amount of reactant or product, an extensive property that is directly proportional to the size of the compartment in which the reaction events occur. This means that the rates of reactions that occur in a 3dimensional compartment are proportional to the volume of the compartment, while the rates of transfers over a 2-dimensional compartment boundary or interface between compartments are proportional to the area of the boundary. Transfer rates are often incorrectly scaled with a volume instead of an area, and the reasons why this is wrong are extensively discussed. I also show how 'textbook' rate equations, which I term canonical rate equations, should be modified for compartmental modelling and how they should be incorporated into either amount-change or concentration-change ODEs.

\section{Introduction}

Multi-compartment models are used in many fields including physiology, pharmacokinetics, epidemiology, biomedicine, systems theory, complexity theory, engineering, physics, information science and social science. There are a plethora of books and papers on the subject, and there will be no attempt in this paper to review them. Nevertheless, there is to my knowledge no published comprehensive treatment of kinetic modelling of compartmentalised reaction networks in the context of systems biology, although some systems biology textbooks [1, 2] do touch on the subject. In my experience there is still a lot of confusion about how to go about constructing such models, and many published models are flawed with respect to how they handle compartmentation. The theory described in this paper arose from our attempts to model compartmentalised reaction networks with variable compartment sizes, and from discussions within the Systems Biology Markup Language (SBML) community about how to describe reaction rate laws in such a way that they need to be specified only once. This paper describes a modelling framework that addresses this issue by answering two questions: Which rate laws should we use to describe the rates of reactions in compartmentalised systems? How should we incorporate these rate laws in the ordinary differential equations that describe the dynamics of the compartmentalised system? 
The "traditional" reaction rate laws found in most physical chemistry, biochemistry and biology textbooks are based on a definition of reaction rate in terms of changes in concentrations of reactants or products per unit time, and are based on the assumptions that (1) all reacting substances are co-located in a single compartment, (2) the compartment is a three-dimensional volume, and (3) the volume size does not vary with time. When defining multi-compartment models the first, and in many cases, the second and third assumptions do not hold. Nevertheless, it would be very useful if these textbook rate laws could still be used in the description of multi-compartment models. I shall show that this is possible once reaction rate is defined in such a way that it handles the following complications:

1. Complex stoichiometry. Compare a reaction with one-to-one stoichiometry, such as $\mathrm{A}+\mathrm{B} \rightarrow \mathrm{C}$, with a reaction with complex stoichiometry, such as $2 \mathrm{~A}+3 \mathrm{~B} \rightarrow 5 \mathrm{C}$. In the simple case the reactant and product concentrations all change at the same rate, namely

$$
-\frac{d a}{d t}=-\frac{d b}{d t}=\frac{d c}{d t},
$$

where $a, b$ and $c$ are the concentrations of A, B and C. Here rate could be defined unambiguously in terms of concentrations changes in any of the three species. In the complex case, however, they all change at different rates. If for the complex case reaction rate were to be defined solely in terms of a time derivative, the value of the rate would depend on which of the $\mathrm{A}, \mathrm{B}$ or $\mathrm{C}$ time derivatives were chosen. This is clearly unsatisfactory: the definition of reaction rate should yield only one value, independent of the choice of time derivative. In this particular example, the only definition that gives the same value no matter which reactant or product is chosen as reference is

$$
-\frac{1}{2} \frac{d a}{d t}=-\frac{1}{3} \frac{d b}{d t}=\frac{1}{5} \frac{d c}{d t},
$$

In none of the three expressions does the rate defined in this way equate to any of the reactant or product time derivatives on their own.

2. Compartmentation. Consider the transfer process $\mathrm{X}_{\text {compt. } 1} \rightleftharpoons \mathrm{X}_{\text {compt. } 2 \text { of a com- }}$ pound $\mathrm{X}$ between two compartments with unequal volumes $V_{1}$ and $V_{2}$. As shown in Section 4, the only way of defining a transfer rate in terms of concentration changes that gives the same value no matter which compartment is referred to is

$$
-V_{1} \frac{d x_{\text {compt. } 1}}{d t}=V_{2} \frac{d x_{\text {compt. } 2}}{d t},
$$

where $x_{\text {compt. } 1}$ and $x_{\text {compt. } 2}$ refer to the concentrations of $\mathrm{X}$ in the two compartments. As with the case of complex reaction stoichiometry, neither concentrationchange time derivative on its own provides an unambiguous value for the rate of transfer. 


\section{Defining reaction and transfer rates}

Both these complications are addressed by using the fundamental definition of reaction rate in terms of the degree of advancement or extent of a reaction (the symbol $\xi$ is commonly used for this quantity) [3]. I prefer to use the associated concept of reaction event or reaction instance as foundation for the definition of reaction rate, as it is much easier to explain. In one reaction event a stoichiometric number of reactant molecules are converted into a stoichiometric number of product molecules. For example, in one event of the reaction $2 \mathrm{~A}+3 \mathrm{~B} \rightarrow 5 \mathrm{C}$, two molecules of $\mathrm{A}$ react with three molecules of $B$ to form five molecules of $C$. It is good practice [3] to distinguish between the stoichiometric coefficients 2, 3, and 5, which are the positive numbers written in front of atoms, ion and molecules in a chemical reaction to balance the number of each element on the reactant and product sides of the equation, and their associated stoichiometric numbers, which are negative for reactants and positive for products, e.g., $-2,-3$, and +5 for $\mathrm{A}, \mathrm{B}$ and $\mathrm{C}$. For any reaction, a forward direction must of course be assigned in order to designate which species are reactants and which are products.

The rate, $v$, of a chemical reaction can now be unambiguously defined as the number of reaction events per time, expressed as moles of reaction events per time, a typical unit being $\mathrm{mol} \mathrm{s}^{-1}$ :

$$
v=\frac{d \xi}{d t}
$$

where $d \xi$, the number of reaction events that occur during time $d t$, can be equated with the degree or extent to which the reaction has advanced during $d t$. Note that throughout this text the symbol $v$ consistently refers to a rate with a unit of mole per time, and never to a rate with unit concentration per time, which is an entity derived from the fundamental definition of reaction rate in eqn. 4.

The number of molecules of substance $X$ that are consumed or produced during one reaction event depends on the stoichiometry with which $X$ participates in the reaction as either a reactant or product. The rate of change in mole amount $n_{\mathrm{X}}$ is therefore related to the reaction rate as defined in eqn. 4 as follows:

$$
\frac{d n_{X}}{d t}=c \cdot \frac{d \xi}{d t}=c v
$$

where $c$ is the stoichiometric number for $X$. Note that for substances $X, Y, Z, \ldots$ the symbols $n_{\mathrm{X}}, n_{\mathrm{Y}}, n_{\mathrm{Z}}, \ldots$ denote their mole amounts while $x, y, z, \ldots$ denote their concentrations.

It follows from eqn. 5 that reaction rate can be expressed in terms of the stoichiometric number and the change per time in the mole amount $n_{\mathrm{X}}$ of a reactant or product $\mathrm{X}$ :

$$
v=\frac{d \xi}{d t}=\frac{1}{c} \frac{d n_{\mathrm{X}}}{d t} \quad\left(\mathrm{mols}^{-1}\right)
$$


Although the definition of reaction rate in terms of a change in substance amount per time instead of a change in concentration per time does not allow the rate of reactions with complex stoichiometry such as $2 \mathrm{~A}+3 \mathrm{~B} \rightarrow 5 \mathrm{C}$ to be expressed by a single mole-amount time derivative on its own, it is at least now consistent with an unambiguously defined reaction rate:

$$
v=\frac{d \xi}{d t}=-\frac{1}{2} \frac{d n_{\mathrm{A}}}{d t}=-\frac{1}{3} \frac{d n_{\mathrm{B}}}{d t}=\frac{1}{5} \frac{d n_{\mathrm{C}}}{d t}
$$

Dividing by the size of the compartment in which $X$ occurs, which is usually a volume of a three-dimensional compartment (but could also be the area of a twodimensional compartment such as a membrane, or the length of a common interface between three compartments), converts the unit of rate from mole amount per time to concentration per time (and $d \xi$ to the number of reaction events per unit size of the compartment, i.e., the concentration of reaction events): ${ }^{1}$

$$
\frac{v}{\text { compartment size }}=\frac{1}{c} \frac{d x}{d t} \quad\left(\text { mol sizeunit }{ }^{-1} \mathrm{~s}^{-1}\right),
$$

where the unit of compartment size could be $\mathrm{dm}^{3}, \mathrm{dm}^{2}$ or $\mathrm{dm}$. Rate expressed in this form is a derived, intensive property that does not depend on the size of the compartment in which the reaction events occur.

The reaction event concept, $\xi$, can be broadened to include transfer events between compartments. In this context $d \xi$ in eqn. 4 refers to the number of transfer events that occur during time $d t$. The definition of transfer rate in terms of a change in substance amount per time instead of a change in concentration per time avoids the complication caused by compartmentation described by eqn. 3 :

$$
v=\frac{d \xi}{d t}=-\frac{d n_{\mathrm{X}_{\text {compt. } 1}}}{d t}=\frac{d n_{\mathrm{X}_{\text {compt. } 2}}}{d t} .
$$

Note again that this is the fundamental definition of transfer rate, and that eqn. 3 is derived from it.

As said in the Introduction, reaction rates in single compartment kinetic models are traditionally defined in terms of substance concentration per time as in eqn. 8, e.g., in $\mathrm{moldm} \mathrm{m}^{-3} \mathrm{~s}^{-1}$ (or, using $\mathrm{M}$ for $\mathrm{mol} \mathrm{dm}^{-3}$ as I occasionally do in this document, $\mathrm{M} \mathrm{s}^{-1}$ ). I shall refer to the textbook rate laws from which these reaction rates are calculated as canonical rate laws and symbolise them with $\mathcal{R}$. My first aim is to show how these canonical rate laws need to be modified for compartmental modelling. Furthermore, it would be advantageous if rate laws need to be specified only once for their use in the ordinary differential equations (ODEs) for different substances in different compartments the sizes of which can vary, whether the ODES are cast as

\footnotetext{
${ }^{1}$ The use of time derivatives to describe rates of change of substance concentrations implicitly assumes that the compartments in which the substances occur are well stirred, i.e., there is no spatial variation in concentration within a compartment.
} 
A.

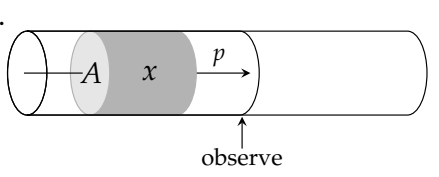

B.

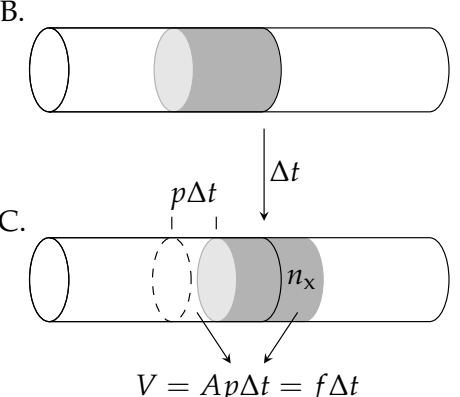

Fig. 1 Visualising the concept of clearance. A. A band of solute $\mathrm{X}$ at concentration $x$ mol dm ${ }^{-3}$ flows at speed $p \mathrm{dm} \mathrm{s}^{-1}$ down a pipe with inner cross-sectional area $A \mathrm{dm}^{2}$. Assume that the boundaries of the band remain sharp, i.e., that there is no diffusion of $X$ into the surrounding pure solvent. B. The front of the band arrives at the observation point. C. After $\Delta t$ seconds the band has moved a distance $p \Delta t \mathrm{dm}$ past the observation point; this corresponds to a volume of $V=A p \Delta t \mathrm{dm}^{3}$, which is also the volume that has been cleared of $\mathrm{X}$ during $\Delta t$ seconds. $V$ contains $n_{\mathrm{X}}=A p \Delta t \cdot x$ mol, the amount of $\mathrm{X}$ that has crossed the observation point during $\Delta t$.

concentration-per-time derivatives or as amount-per-time derivatives. It turns out that the solution to the first question is very simple and follows from the fact that the fundamental substance amount-change rate as defined in eqn. 6 is an extensive property that is proportional to the size of the compartment in which the reaction events occur. ${ }^{2}$ This implies that the canonical rate law $\mathcal{R}$ should be scaled by multiplying with the size of the compartment with which the reaction or transfer events are associated, and that the units of the rate constants should be consistent with mol s${ }^{-1}$ rates. ${ }^{3}$ To explain this convincingly I need the concept of clearance.

\section{Clearance}

Clearance is a crucial physiological concept that co-determines the rate of transfer of a solute between compartments. In physiology and pharmacokinetics, clearance is the volume of plasma from which a substance is completely removed per unit time. A simple way of visualising clearance is to consider a band of solute $\mathrm{X}$ with concentration $x$ mol dm${ }^{-3}$ flowing down a pipe with inner cross-sectional area $A \mathrm{dm}^{2}$ at a

\footnotetext{
${ }^{2}$ If $x$ reaction events occur per unit time in a compartment, then $0.5 x$ reaction events will occur per unit time in half of the compartment, i.e., the rate $v$ will be half of that in the full compartment.

${ }^{3}$ Such scaled rate laws are the "kinetic laws" that the Systems Biology Markup Language (SBML) [4] refers to.
} 


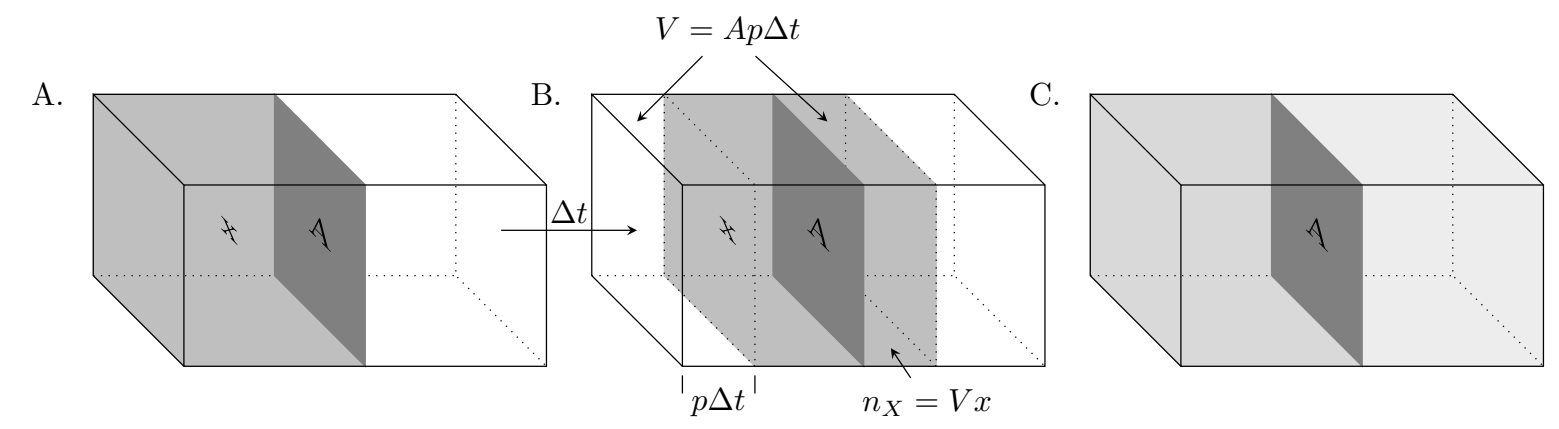

Fig. 2 Diffusion across a membrane separating two compartments. A.Two compartments are separated by a membrane with area $A \mathrm{dm}^{2}$ and permeability $p \mathrm{dm} \mathrm{s}^{-1}$ for solute $\mathrm{X}$. The left-hand compartment initially contains $\mathrm{X}$ at concentration $x \mathrm{moldm}^{-3}$, while the right-hand compartment contains no X. B. Thought experiment: imagine that during time interval $\Delta t$ seconds an amount $n_{\mathrm{X}}$ mol diffuses through the membrane in such a way that the concentration of $\mathrm{X}$ on both sides of the membrane remains constant. This means that in the left-hand compartment a volume $V=A p \Delta t \mathrm{dm}^{3}$ must be cleared of $\mathrm{X}$, while in the right-hand compartment $n_{\mathrm{X}}$ must be confined to the same volume $V$. As in Fig. 1, $n_{\mathrm{X}}=A p \Delta t \cdot x$ and the rate of transfer over the membrane is $n_{\mathrm{X}} / \Delta t=A p \cdot x$. C. In reality $\mathrm{X}$ is of course uniformly distributed in each compartment so that during time interval $\Delta t$ the concentration in left-hand compartment decreases while that in the right-hand compartment increases (see text).

constant speed of $p \mathrm{dm} \mathrm{s}^{-1}$. Fig. 1 describes how to calculate the mole amount $n_{\mathrm{X}}$ of solute that crosses an observation point during a given time interval $\Delta t$ as

$$
n_{\mathrm{X}}=A p \cdot \Delta t \cdot x \mathrm{~mol}
$$

The amount-change rate at which $\mathrm{X}$ has moved past the boundary is therefore

$$
\frac{n_{\mathrm{X}}}{\Delta t}=A p \cdot x=f x \mathrm{mols}^{-1},
$$

where $f=A p$ is the clearance with unit $\mathrm{dm}^{3} \mathrm{~s}^{-1}$, the volume cleared of $\mathrm{X}$ per unit time. Since in compartmental modelling $f$ will always occur in the context of rate equations I shall from here on refer to it as the rate factor. Note that whereas $p$ is a kinetic constant, $f$ varies with $A$, and it is for this reason that I describe it as a factor in the rate equation and not as a constant.

\section{Transfer rates between compartments}

Fig. 2 explains clearance in the context of diffusion of a solute over a membrane between two compartments. The situation in Figs. $2 \mathrm{~A}$ and $\mathrm{B}$ is analogous to that in Figs. $1 \mathrm{~B}$ and $\mathrm{C}$, but the reality is of course that of Fig. $2 \mathrm{C}$ where solute $\mathrm{X}$ can cross 
the membrane in both directions. The net rate of transfer from the lefthand to the righthand compartment is

$$
v=-\frac{d n_{X_{\mathrm{L}}}}{d t}=\frac{d n_{\mathrm{X}_{\mathrm{R}}}}{d t}=f_{\mathrm{f}} x_{\mathrm{L}}-f_{\mathrm{r}} x_{\mathrm{R}}=A\left(p_{\mathrm{f}} x_{\mathrm{L}}-p_{\mathrm{r}} x_{\mathrm{R}}\right),
$$

where the subscripts $\mathrm{L}$ and $\mathrm{R}$ refer to the left-hand and right-hand compartments, and $p_{\mathrm{f}}$ and $p_{\mathrm{r}}$ are now properly seen to be permeability constants in the forward and reverse directions with unit $\mathrm{dms}^{-1}$. Similarly, $f_{\mathrm{f}}$ and $f_{\mathrm{r}}$ are the rate factors in the forward and reverse directions with unit $\mathrm{dm}^{3} \mathrm{~s}^{-1}$. Note that $p_{\mathrm{f}}$ and $p_{\mathrm{r}}$ are kinetic constants similar to the rate constants $k_{\mathrm{f}}$ and $k_{\mathrm{r}}$ in mass-action rate equations for chemical reactions; they differ only in their units. The rate factors $f_{\mathrm{f}}$ and $f_{\mathrm{r}}$, however, depend on the area of the membrane that separates the compartments, which can be a system variable.

The first lesson to be learnt here is that the amount-change rate of transfer across the membrane is directly proportional to the area of the interface between the compartments, i.e., to the size of the two-dimensional "compartment" with which the transfer events are associated. It is never, as is often claimed, proportional to the volume of one of the compartments (see Section 10 for an exhaustive explanation).

The transfer process $\mathrm{X}_{\mathrm{L}} \rightleftharpoons \mathrm{X}_{\mathrm{R}}$ is analogous to the single-compartment mass-action chemical reaction $\mathrm{X} \rightleftharpoons \mathrm{Y}$. The canonical concentration-change rate law $\mathcal{R}$ for the chemical reaction is

$$
-\frac{d x}{d t}=\frac{d y}{d t}=k_{\mathrm{f}} x-k_{\mathrm{r}} y
$$

with the rate constants in $s^{-1}$. Here is the first instance of the protocol for transforming a canonical rate equation into the appropriate amount-change rate equation (eqn. 12): Multiply with the size of the compartment with which the transfer events are associated (here the area of the two-dimensional membrane that separates the compartments) and adjust the units of the rate constants (here from s$~^{-1}$ to $\mathrm{dm} \mathrm{s}^{-1}$ ) so that the rate is expressed in $\mathrm{mol} \mathrm{s}^{-1}$.

In the more common situation where the permeability constant $p$ is the same in both directions, rate eqn. 12 simplifies to

$$
v=f\left(x_{\mathrm{L}}-x_{\mathrm{R}}\right)=A \cdot p\left(x_{\mathrm{L}}-x_{\mathrm{R}}\right) \text {. }
$$

To obtain concentration-change rate equations each time derivative has to be divided by the corresponding volume:

$$
-\frac{1}{V_{\mathrm{L}}} \frac{d n_{\mathrm{X}_{\mathrm{L}}}}{d t}=-\frac{d x_{\mathrm{L}}}{d t}=\frac{1}{V_{\mathrm{L}}}\left(f_{\mathrm{f}} x_{\mathrm{L}}-f_{\mathrm{r}} x_{\mathrm{R}}\right)=\frac{A}{V_{\mathrm{L}}}\left(p_{\mathrm{f}} x_{\mathrm{L}}-p_{\mathrm{r}} x_{\mathrm{R}}\right)=\frac{A}{V_{\mathrm{L}}} \mathcal{R}
$$

and

$$
\frac{1}{V_{\mathrm{R}}} \frac{d n_{\mathrm{X}_{\mathrm{R}}}}{d t}=\frac{d x_{\mathrm{R}}}{d t}=\frac{1}{V_{\mathrm{R}}}\left(f_{\mathrm{f}} x_{\mathrm{L}}-f_{\mathrm{r}} x_{\mathrm{R}}\right),=\frac{A}{V_{\mathrm{R}}}\left(p_{\mathrm{f}} x_{\mathrm{L}}-p_{\mathrm{r}} x_{\mathrm{R}}\right)=\frac{A}{V_{\mathrm{R}}} \mathcal{R}
$$


from which it follows that, as in eqn. 3,

$$
-V_{\mathrm{L}} \frac{d x_{\mathrm{L}}}{d t}=V_{\mathrm{R}} \frac{d x_{\mathrm{R}}}{d t}
$$

The second lesson is that the only difference between amount-change and concentration-change transfer rate equations is the scaling factor (area versus area/volume). The canonical part of the rate law $\left(p_{\mathrm{f}} x_{\mathrm{L}}-p_{\mathrm{r}} x_{\mathrm{R}}\right)$ is the same for both.

The above two lessons will be seen to hold in general, also for chemical reactions that occur at compartmental interfaces, which I shall treat after considering how compartmental geometry affects transfer rates.

\section{Compartmental geometry}

Compartmental geometry is an important factor in compartmental modelling. Consider the geometries in Fig. 3 and assume that in the following comparisons the concentration $x_{1}$ in compartment 1 is the same for all four configurations, and likewise $x_{2}$ in compartment 2 .

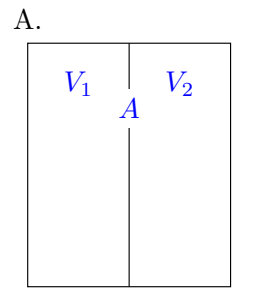

B.
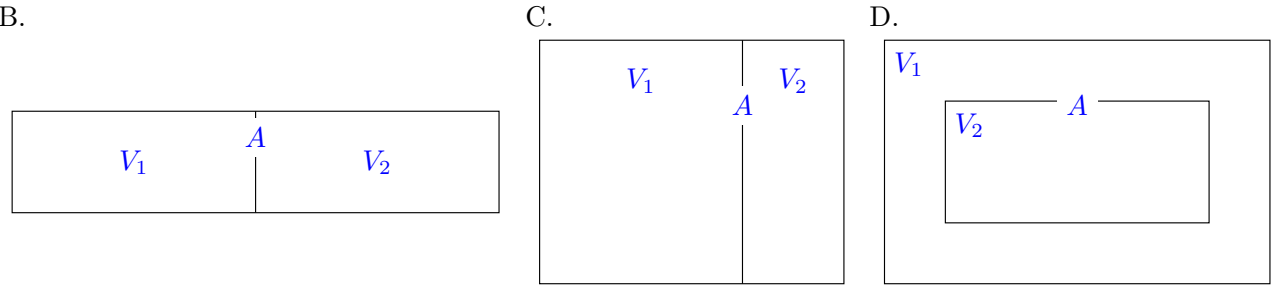

Fig. 3 Different compartmental configurations.

1. In Fig. $3 \mathrm{~A}$ and B the compartment volumes are the same, but the interface area $A$ in Fig. $3 \mathrm{~A}$ is larger than that in $\mathrm{B}$, so both the amount-change and the concentration-change transfer rates will therefore be proportionally faster in A.

2. In Fig. $3 \mathrm{~A}$ and $\mathrm{C}$, on the other hand, the interface area $A$ is the same, but whereas in A the volumes are equal, in $C V_{1}=2 V_{2}$. This means that whereas the amountchange transfer rate is the same in both cases, the concentration-change transfer rates are not: while in Fig. $3 \mathrm{~A}-d x_{1} / d t=d x_{2} / d t$, in Fig. $3 \mathrm{C}-d x_{1} / d t=$ $0.5 d x_{2} / d t$.

3. Fig. 3D has the geometry of one compartment inside another. $V_{1}$ can change without affecting $V_{2}$ and $A$; assuming that $x_{1}$ remains constant, such a change 
in $V_{1}$ will affect neither the amount-change transfer rate nor the concentrationchange transfer rate $d x_{2} / d t$, but will affect $-d x_{1} / d t$, which is inversely proportional to $V_{1}$. A change in $V_{2}$ will affect both $V_{1}$ and $A$, and thereby also the amount-change transfer rate (through the change in $A$ ) and both concentrationchange transfer rates: $-d x_{1} / d t$ (through the change in $A$ and $V_{1}$ ) and $d x_{2} / d t$ (through the changes in $A$ and $V_{2}$ ).

What if the size of the compartment with which the transfer events are associated (the area $A$ ) is unknown? Here the correct way of specifying the rate equation is by using rate factors, as in the amount-change transfer rate equation

$$
v=-\frac{d n_{\mathrm{X}_{1}}}{d t}=\frac{d n_{\mathrm{X}_{2}}}{d t}=f_{\mathrm{f}} x_{1}-f_{\mathrm{r}} x_{2}
$$

or in the concentration-change transfer rate equations ${ }^{4}$

$$
-\frac{d x_{1}}{d t}=\frac{1}{V_{1}}\left(f_{\mathrm{f}} x_{1}-f_{\mathrm{r}} x_{2}\right) \quad \text { and } \quad \frac{d x_{2}}{d t}=\frac{1}{V_{2}}\left(f_{\mathrm{f}} x_{1}-f_{\mathrm{r}} x_{2}\right) .
$$

\section{Interlude: A thought experiment}

Consider a reversible chemical reaction $\mathrm{X}+\mathrm{Y} \rightleftharpoons \mathrm{Z}$ with the canonical mass-action concentration-change rate law

$$
-\frac{d x}{d t}=-\frac{d y}{d t}=\frac{d z}{d t}=k_{\mathrm{f}} x y-k_{\mathrm{r}} z=\mathcal{R} .
$$

with $k_{\mathrm{f}}$ in $\mathrm{moldm} \mathrm{dm}^{-3} \mathrm{~s}^{-1}$ and $k_{\mathrm{r}}$ in $\mathrm{s}^{-1}$.

When the reaction occurs in a single compartment with volume $V_{1}$ (Fig. 4A) the amount-change rate is

$$
v=V_{1}\left(k_{\mathrm{f}} \frac{n_{\mathrm{X}}}{V_{1}} \frac{n_{\mathrm{Y}}}{V_{1}}-k_{\mathrm{r}} \frac{n_{\mathrm{Z}}}{V_{1}}\right)=V_{1} \mathcal{R}
$$

For the purposes of the thought experiment the concentrations are explicitly written as mole amount over volume to indicate with respect to which compartment size each concentration is defined.

Now consider $\mathrm{Y}$ and $\mathrm{Z}$ to be confined to a smaller volume $V_{2}$ (the grey reacting zone in Fig. 4B), while $X$ still occupies the whole of $V_{1}$. There is no physical boundary between the grey and white zones (remember that this is a thought experiment!). In Fig. $4 \mathrm{~A}$ the reaction events occurred throughout $V_{1}$, so that the amount-change rate was proportional to $V_{1}$. By contrast, in Fig. $4 \mathrm{~B}$ the reaction events occur only in

\footnotetext{
${ }^{4}$ Section 10 explains why the often used $\frac{d x_{1}}{d t}=-k_{\mathrm{f}} x_{1}+k_{\mathrm{r}} x_{2} \cdot \frac{V_{2}}{V_{1}}$ and $\frac{d x_{2}}{d t}=\frac{V_{1}}{V_{2}} \cdot k_{\mathrm{f}} x_{1}-k_{\mathrm{r}} x_{2}$ is wrong.
} 
A.

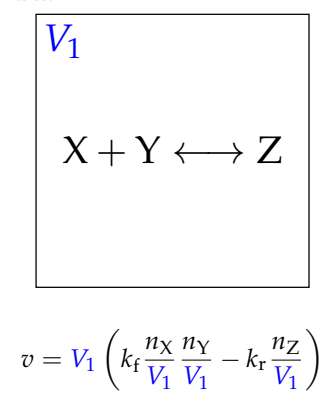

B.

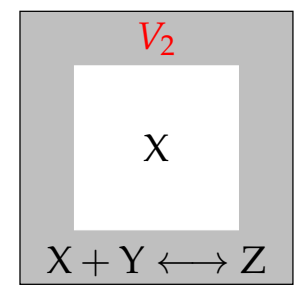

$v=V_{2}\left(k_{\mathrm{f}} \frac{n_{\mathrm{X}}}{V_{1}} \frac{n_{\mathrm{Y}}}{V_{2}}-k_{\mathrm{r}} \frac{n_{\mathrm{Z}}}{V_{2}}\right)$
C.

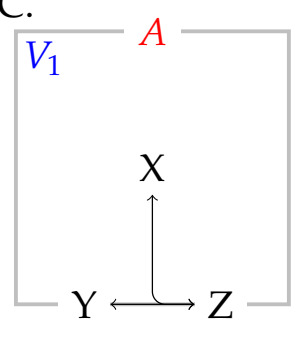

$v=A\left(k_{\mathrm{f}} \frac{n_{\mathrm{X}}}{V_{1}} \frac{n_{\mathrm{Y}}}{A}-k_{\mathrm{r}} \frac{n_{\mathrm{Z}}}{A}\right)$

Fig. 4 An amount-change rate is proportional to the size of the compartment in which the reaction events occur. A. A reversible reaction $\mathrm{X}+\mathrm{Y} \rightleftharpoons \mathrm{Z}$ takes place in a 3-D compartment with volume $V_{1}$. B. Y and $Z$ are now confined to the grey reacting zone with volume $V_{2}$, while $\mathrm{X}$ still occupies the whole of $V_{1}$. C. The reacting zone with volume $V_{2}$ has decreased to the limit where it becomes a 2-D compartment the size of which is the area $A$ of the $V_{1}$-compartment boundary. The rate equations are discussed in the text.

$V_{2}$ where $\mathrm{X}$ and $\mathrm{Y}$ are in direct contact, so that the rate $v$ is now proportional to $V_{2}$ instead of $V_{1}$ :

$$
v=V_{2}\left(k_{\mathrm{f}} \frac{n_{\mathrm{X}}}{V_{1}} \frac{n_{\mathrm{Y}}}{V_{2}}-k_{\mathrm{r}} \frac{n_{\mathrm{Z}}}{V_{2}}\right)=V_{2} \mathcal{R} .
$$

When the reacting zone $V_{2}$ decreases to the limit it becomes an idealised 2-D compartment, the size of which is the area, $A$, of the $V_{1}$-compartment boundary (Fig. $4 \mathrm{C}$ ). $\mathrm{Y}$ and $\mathrm{Z}$ are now confined to this 2-D compartment with concentrations $y=n_{\mathrm{Y}} / A$ and $z=n_{\mathrm{Z}} / A$, which means that the reaction events are associated with the boundary and the rate $v$ is proportional to the boundary area $A$ :

$$
v=A\left(k_{\mathrm{f}} \frac{n_{\mathrm{X}}}{V_{1}} \frac{n_{\mathrm{Y}}}{A}-k_{\mathrm{r}} \frac{n_{\mathrm{Z}}}{A}\right)=A \mathcal{R} .
$$

All the rate equations can be also expressed using rate factors. The rate factors for the forward rate component of the three rate equations would be $k_{\mathrm{f}} V_{1}, k_{\mathrm{f}} V_{2}$, and $k_{\mathrm{f}} A$ and for the reverse rate component $k_{\mathrm{r}} V_{1}, k_{\mathrm{r}} V_{2}$, and $k_{\mathrm{r}} A$. The unit of $k_{\mathrm{f}}$ and $k_{\mathrm{r}}$ in the rate factors that contain $V_{1}$ and $V_{2}$ is s ${ }^{-1}$, while in the rate factor that contains $A$ it is $\mathrm{dm}^{3} \mathrm{~s}^{-1}$.

Again, as in the previous section, the amount-change rate equations differ only in terms of the size factor that scales the canonical rate law $\mathcal{R}=k_{\mathrm{f}} x y-k_{\mathrm{r}} z$. As before, the scaling factor is the size of the compartment with which the reaction events are associated.

\section{Reactions at the interface between compartments}

Let us now consider that the chemical reaction $X+Y \rightleftharpoons Z$ takes place at the interface between compartments and that the reactants and products can occur in any of the three compartments with sizes $V_{1}, V_{2}$, and $A$ (Fig. 5). 

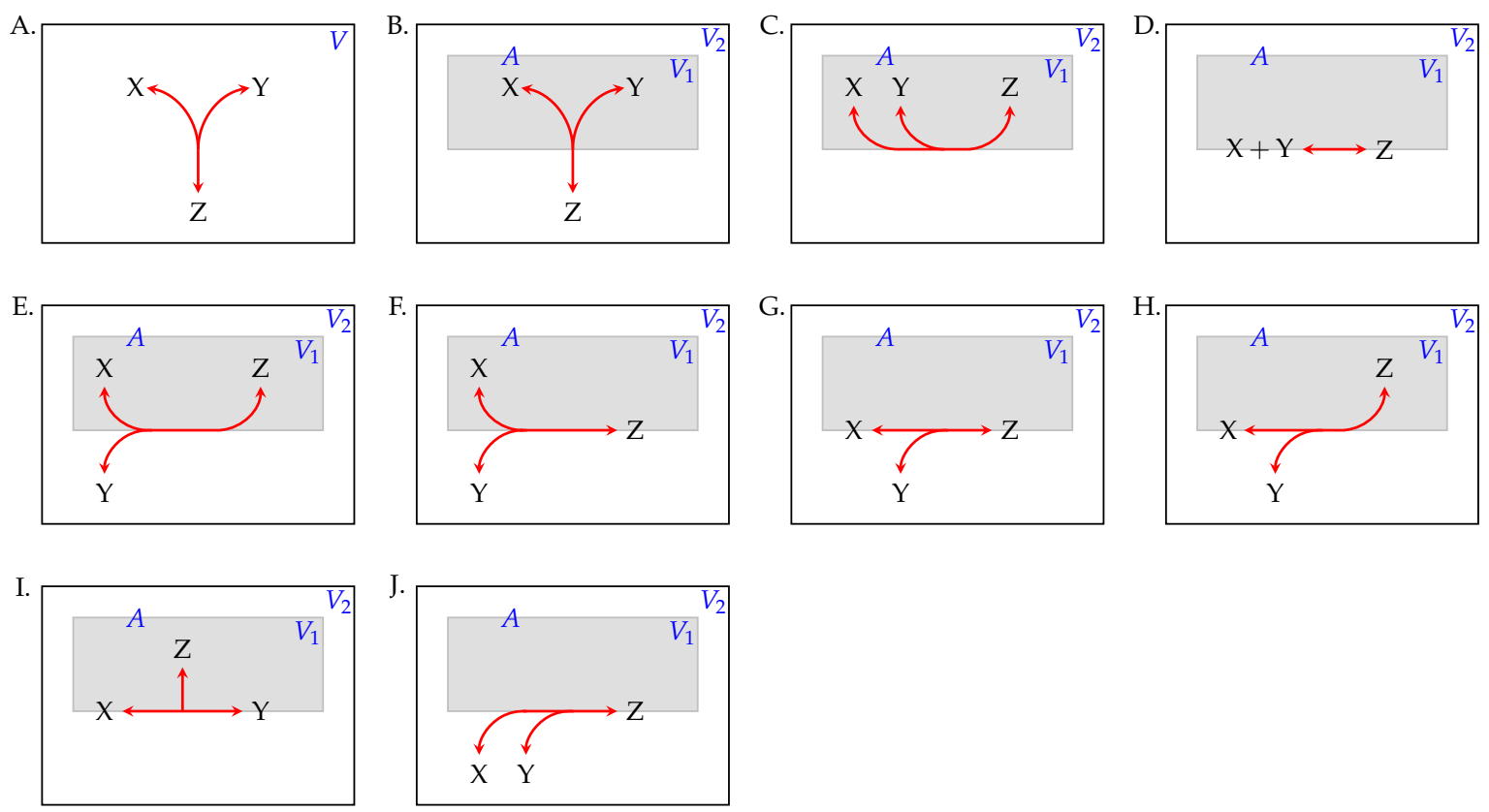

Fig. 5 A bi-uni reaction $\mathrm{X}+\mathrm{Y} \rightleftharpoons \mathrm{Z}$ that $\mathrm{A}$. occurs in a single compartment with volume $V$, or B.-J. takes place in or on the 2-dimensional interface compartment with area $A$ between two 3 -dimensional compartments with volumes $V_{1}$ and $V_{2}$. Where a reagent occurs on the compartmental boundary it implies that it is confined to the boundary and does not diffuse into either of the two compartments.

Note that in general the interface need not be a physical boundary such as a membrane, but could just be a boundary where two immiscible phases meet: solutes from both phases are in contact and can react at this interphase boundary. There are many hypothetical configurations for reactions that take place in the interface (for the purposes of this discussion it is immaterial whether they are realistic). Fig. 5B-J shows a representative sample of such putative configurations (the single compartment reaction in Fig. 5A is included for comparative purposes).

1. Reactants and products are separated by the interface at which the chemical reaction occurs (Fig. 5B);

2. Reactants and products reside in the same compartment volume but the reaction takes place on the compartment boundary (Fig. 5C);

3. All reactants and products reside within the 2-dimensional interface (Fig. 5D);

4. Reactants reside in separate compartment volumes and react at the interface to form a product into either a compartment volume (Fig. 5E) or the interface (Fig. 5F);

5. Some reactants or products reside in the interface while one or both of the 3dimensional compartments contain the others (Fig. 5G -J); 
The amount-change rate equations for the compartmental configurations in Fig. 5 are listed in Table 1, together with the units of the rate constants $k_{\mathrm{f}}$ and $k_{\mathrm{r}}$.

Table 1 Amount-change rate equations and rate constant units for the configurations in Fig. 5. Concentrations are explicitly written as mole/compartment size to indicate with respect to which compartment the concentrations are defined. The symbol $\mathrm{M}$ signifies $\mathrm{mol} \mathrm{dm}^{-3}$. In all cases the canonical rate equation is $\mathcal{R}=k_{\mathrm{f}} x y-k_{\mathrm{r}} z$.

\begin{tabular}{|c|c|c|c|c|}
\hline & $v$ & $k_{\mathrm{f}}$ & $k_{\mathrm{r}}$ & $k_{\mathrm{f}}$ (basic units) \\
\hline A. & $V \cdot\left(k_{\mathrm{f}} \frac{n_{\mathrm{X}}}{V} \frac{n_{\mathrm{Y}}}{V}-k_{\mathrm{r}} \frac{n_{\mathrm{Z}}}{V}\right)$ & $\mathrm{M}^{-1} \mathrm{~s}^{-1}$ & $\mathrm{~s}^{-1}$ & $\mathrm{dm}^{3} \mathrm{~mol}^{-1} \mathrm{~s}^{-1}$ \\
\hline B. & $A \cdot\left(k_{\mathrm{f}} \frac{n_{\mathrm{X}}}{V_{1}} \frac{n_{\mathrm{Y}}}{V_{1}}-k_{\mathrm{r}} \frac{n_{\mathrm{Z}}}{V_{0}}\right)$ & $\mathrm{dm} \mathrm{M} \mathrm{M}^{-1} \mathrm{~s}^{-1}$ & $\mathrm{dm} \mathrm{s}^{-1}$ & $\mathrm{dm}^{4} \mathrm{~mol}^{-1} \mathrm{~s}^{-1}$ \\
\hline C. & $A \cdot\left(k_{\mathrm{f}} \frac{n_{\mathrm{X}}}{V_{1}} \frac{n_{\mathrm{Y}}}{V_{1}}-k_{\mathrm{r}} \frac{n_{\mathrm{Z}}}{V_{1}}\right)$ & $\mathrm{dm} \mathrm{M}^{-1} \mathrm{~s}^{-1}$ & $\mathrm{dm} \mathrm{s}^{-1}$ & $\mathrm{dm}^{4} \mathrm{~mol}^{-1} \mathrm{~s}^{-1}$ \\
\hline D. & $A \cdot\left(k_{\mathrm{f}} \frac{n_{\mathrm{X}}}{A} \frac{n_{\mathrm{Y}}}{A}-k_{\mathrm{r}} \frac{n_{\mathrm{Z}}}{A}\right)$ & $\mathrm{dm}^{2} \mathrm{~mol}^{-1} \mathrm{~s}^{-1}$ & $\mathrm{~s}^{-1}$ & $\mathrm{dm}^{2} \mathrm{~mol}^{-1} \mathrm{~s}^{-1}$ \\
\hline E. & $A \cdot\left(k_{\mathrm{f}} \frac{n_{\mathrm{X}}}{V_{1}} \frac{n_{\mathrm{Y}}}{V_{2}}-k_{\mathrm{r}} \frac{n_{\mathrm{Z}}}{V_{1}}\right)$ & $\mathrm{dm} \mathrm{M}^{-1} \mathrm{~s}^{-1}$ & $\mathrm{dm} \mathrm{s}^{-1}$ & $\mathrm{dm}^{4} \mathrm{~mol}^{-1} \mathrm{~s}^{-1}$ \\
\hline F. & $A \cdot\left(k_{\mathrm{f}} \frac{n_{\mathrm{X}}}{V_{1}} \frac{n_{\mathrm{Y}}}{V_{2}}-k_{\mathrm{r}} \frac{n_{\mathrm{Z}}}{A}\right)$ & $\mathrm{dm} \mathrm{M}^{-1} \mathrm{~s}^{-1}$ & $\mathrm{~s}^{-1}$ & $\mathrm{dm}^{4} \mathrm{~mol}^{-1} \mathrm{~s}^{-1}$ \\
\hline G. & $A \cdot\left(k_{\mathrm{f}} \frac{n_{\mathrm{X}}}{A} \frac{n_{\mathrm{Y}}}{V_{2}}-k_{\mathrm{r}} \frac{n_{\mathrm{Z}}}{A}\right)$ & $\mathrm{M}^{-1} \mathrm{~s}^{-1}$ & $\mathrm{~s}^{-1}$ & $\mathrm{dm}^{3} \mathrm{~mol}^{-1} \mathrm{~s}^{-1}$ \\
\hline H. & $A \cdot\left(k_{\mathrm{f}} \frac{n_{\mathrm{X}}}{A} \frac{n_{\mathrm{Y}}}{V_{2}}-k_{\mathrm{r}} \frac{n_{\mathrm{Z}}}{V_{1}}\right)$ & $\mathrm{M}^{-1} \mathrm{~s}^{-1}$ & $\mathrm{dm} \mathrm{s}^{-1}$ & $\mathrm{dm}^{3} \mathrm{~mol}^{-1} \mathrm{~s}^{-1}$ \\
\hline I. & $A \cdot\left(k_{\mathrm{f}} \frac{n_{\mathrm{X}}}{A} \frac{n_{\mathrm{Y}}}{A}-k_{\mathrm{r}} \frac{n_{\mathrm{Z}}}{V_{1}}\right)$ & $\mathrm{dm}^{2} \mathrm{~mol}^{-1} \mathrm{~s}^{-1}$ & $\mathrm{dm} \mathrm{s}^{-1}$ & $\mathrm{dm}^{2} \mathrm{~mol}^{-1} \mathrm{~s}^{-1}$ \\
\hline $\mathrm{J}$. & $A \cdot\left(k_{\mathrm{f}} \frac{n_{\mathrm{X}}}{V_{2}} \frac{n_{\mathrm{Y}}}{V_{2}}-k_{\mathrm{r}} \frac{n_{\mathrm{Z}}}{A}\right)$ & $\mathrm{dm} \mathrm{M}^{-1} \mathrm{~s}^{-1}$ & $\mathrm{~s}^{-1}$ & $\mathrm{dm}^{4} \mathrm{~mol}^{-1} \mathrm{~s}^{-1}$ \\
\hline
\end{tabular}

It may seem strange that volume-based and area-based concentrations can be freely mixed in a rate law. However, it becomes obvious that this is correct when one realises that the concentration of a solute in the narrow reaction zone that borders the boundary of a compartment, i.e., that concentration at which the solute is in direct contact with and reacts with solutes confined to the boundary, is precisely the solute concentration in the well-mixed volume compartment as a whole (refer back to the thought experiment in Section 6). The amount $n_{\mathrm{X}_{\mathrm{A}}}$ mol of a solute $\mathrm{X}$ at some surface area $A$ of a compartment with volume $V$ that contains $n_{\mathrm{X}_{\mathrm{V}}}$ mol of $\mathrm{X}$ is $n_{\mathrm{X}_{\mathrm{A}}}=\frac{A}{V} \cdot n_{\mathrm{X}_{\mathrm{V}}}$, i.e., $n_{\mathrm{X}_{\mathrm{A}}} / A=n_{\mathrm{X}_{\mathrm{V}}} / V$.

A last example is provided in Fig. 6. Here the reaction takes place at the interface where three compartments with volumes $V_{1}, V_{2}$, and $V_{3}$ meet, i.e., at the 1dimensional compartment with length $L$ in the centre of the diagram. The amount- 


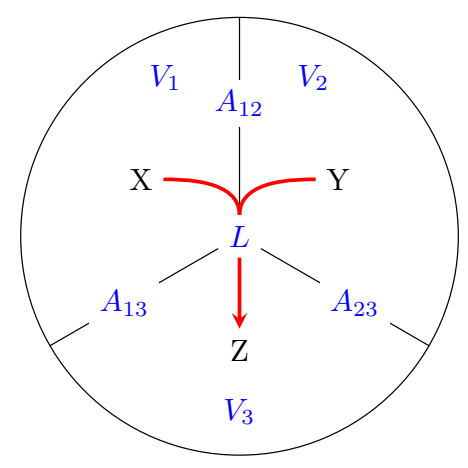

Fig. 6 A reaction $X+Y \rightleftharpoons Z$ that occurs at the 1-dimensional interface with length $L$ where three compartments meet.

change rate law is proportional to $L$ (in $\mathrm{dm}$ ):

$$
v=L \cdot\left(k_{\mathrm{f}} x y-k_{\mathrm{r}} z\right)=L \cdot\left(k_{\mathrm{f}} \frac{n_{\mathrm{X}}}{V_{1}} \frac{n_{\mathrm{Y}}}{V_{2}}-k_{\mathrm{r}} \frac{n_{\mathrm{Z}}}{V_{3}}\right)=L \mathcal{R}
$$

with $k_{\mathrm{f}}$ in $\mathrm{dm}^{2} \mathrm{M}^{-1} \mathrm{~s}^{-1}$ and $k_{\mathrm{r}}$ in $\mathrm{dm}^{2} \mathrm{~s}^{-1}$.

\section{Transfer through pores or by mediator facilitation}

Up to now I have only considered processes that occur over the whole area of a 2dimensional interface between two compartments, i.e., processes such as unaided diffusion through, reaction within, or catalysis by the whole surface. Here the size of the interfacial area matters-the larger the area, the faster the amount change rate.

However, such processes can also occur through pores (channels) or mediators such as transporters or catalysts located in a membrane that separates the compartments. In principle, the number of pores or mediators are independent of the membrane area, in the sense that the area can vary while the number of mediators remains constant and vice versa. Here we shall see that it is the number of the pores or mediators in the interface area that matters, not the size of the area itself. Fig. 7 shows the same compartmental configurations as those in Fig. 3, but now, irrespective of the size of the interfacial area, the number of pores or mediators are the same.

Consider the 2-compartment configurations in Fig. 7 where the transfer of substances does not take place over the whole membrane but only with the help of pores in the membrane or mediator molecules such as transporters or enzymes floating in the membrane. Let the concentration of such a pore or mediator $\mathrm{M}$ in the 2-dimensional membrane with area $A$ be $m=n_{\mathrm{M}} / A$. Just as an enzyme-catalysed reaction rate is proportional to the enzyme concentration, so the concentration-change rate must here be proportional to the mediator or pore concentration, $m$.

As a starting point, consider the simplest possible reversible and non-saturable canonical concentration-change rate law for the mediated reaction $\mathrm{X} \rightleftharpoons \mathrm{Y}$ in a com- 
A.

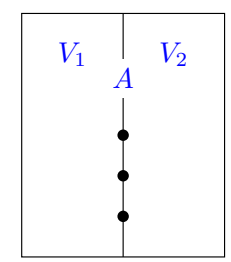

B.

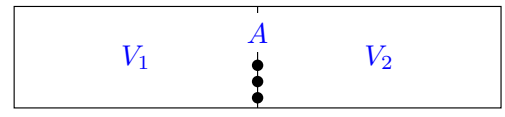

C.

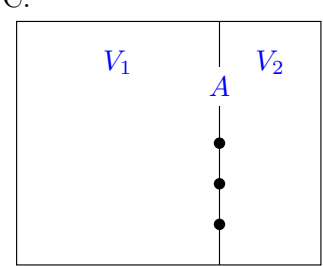

D.

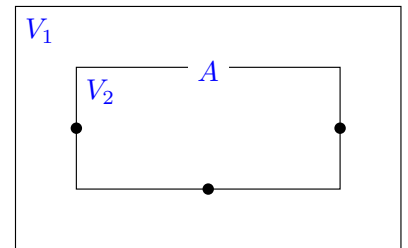

Fig. 7 Different compartmental configurations. The dots represent pores or mediators, which can be channels, transporters, or enzymes.

partment with volume $V$ :

$$
\mathcal{R}=m\left(k_{\mathrm{f}} x-k_{\mathrm{r}} y\right) .
$$

Since $\mathcal{R}$ here refers to single-compartment kinetics, the second-order rate constants $k_{\mathrm{f}}$ and $k_{\mathrm{r}}$ are in $\mathrm{M}^{-1} \mathrm{~s}^{-1}$ and $m=n_{\mathrm{M}} / V$ in $\mathrm{mol} \mathrm{dm} \mathrm{m}^{-3}$.

For the 2-compartment configurations in Fig. 7 the amount-change rate law is obtained, as always, by multiplying $\mathcal{R}$ with the area of the membrane in which the mediator molecules occur:

$$
v=A \cdot m\left(k_{\mathrm{f}} x_{1}-k_{\mathrm{r}} x_{2}\right)
$$

where $x_{1}$ and $x_{2}$ are the concentrations of substance $\mathrm{X}$ in the two compartments. The rate constants $k_{\mathrm{f}}$ and $k_{\mathrm{r}}$ are still in $\mathrm{M}^{-1} \mathrm{~s}^{-1}$ and $m$ is now in $\mathrm{mol} \mathrm{dm}{ }^{-2}$, as required by the fact that the mediator molecules are confined to the 2-dimensional membrane.

Although it appears as if the rate is proportional to the area, it is not. The area cancels from the $A \cdot m=A \cdot n_{\mathrm{M}} / A$ term so that the rate is actually proportional to $n_{\mathrm{M}}$, the number of mediator molecules or the number of pores in the membrane:

$$
v=n_{\mathrm{M}}\left(k_{\mathrm{f}} x_{1}-k_{\mathrm{r}} x_{2}\right)=f_{\mathrm{f}} x_{1}-f_{\mathrm{r}} x_{2},
$$

where $f_{\mathrm{f}}=n_{\mathrm{M}} k_{\mathrm{f}}$ and $f_{\mathrm{r}}=n_{\mathrm{M}} k_{\mathrm{r}}$. Note that although A cancels, the rule that the canonical rate law $\mathcal{R}$ must be multiplied with $A$ still holds.

To obtain concentration-change rate equations, eqn. 27 has to be divided by the corresponding volumes:

$$
-\frac{d x_{1}}{d t}=\frac{n_{\mathrm{M}}}{V_{1}}\left(p_{\mathrm{f}} x_{1}-p_{\mathrm{r}} x_{2}\right)=\frac{1}{V_{1}}\left(f_{\mathrm{f}} x_{1}-f_{\mathrm{r}} x_{2}\right)
$$

and

$$
\frac{d x_{2}}{d t}=\frac{n_{\mathrm{M}}}{V_{2}}\left(p_{\mathrm{f}} x_{1}-p_{\mathrm{r}} x_{2}\right)=\frac{1}{V_{2}}\left(f_{\mathrm{f}} x_{1}-f_{\mathrm{r}} x_{2}\right) .
$$

Although the terms $n_{\mathrm{M}} / V_{1}$ and $n_{\mathrm{M}} / V_{2}$ are in mol dm ${ }^{-3}$ they are not "true" concentrations since $\mathrm{M}$ only occurs in area $A$ and its "true" concentration is $n_{\mathrm{M}} / A$. They can, however, be regarded as "effective" concentrations of $\mathrm{M}$ as seen from the viewpoints of the two compartments: as a compartment volume increases the effective concentration of $\mathrm{M}$ decreases and vice versa. 
In the case of a saturable, facilitated-transport or enzyme-catalysed process the canonical concentration-change rate law is the reversible Michaelis-Menten rate law for the enzyme-catalysed reaction $\mathrm{X} \rightleftharpoons \mathrm{Y}$ :

$$
\mathcal{R}=m\left(\frac{k_{\mathrm{f}} x-k_{\mathrm{r}} y}{1+\frac{x}{K_{\mathrm{X}}}+\frac{y}{K_{\mathrm{Y}}}}\right),
$$

where $m$ is the transporter concentration or the enzyme concentration in $\mathrm{mol} \mathrm{dm}^{-3}$, $k_{\mathrm{f}}=\frac{k_{\mathrm{cat}, \mathrm{f}}}{K_{X}}$ and $k_{\mathrm{r}}=\frac{k_{\mathrm{cat}, \mathrm{r}}}{K_{Y}}$ in $\mathrm{M}^{-1} \mathrm{~s}^{-1}$ are the specificity constants, and $K_{\mathrm{X}}$ and $K_{\mathrm{Y}}$ in mol $\mathrm{dm}^{-3}$ are the substrate and product half-saturation constants.

The associated amount-change rate law for the 2-compartment configuration in Fig. 2C would be

$$
v=A \cdot m\left(\frac{k_{\mathrm{f}} x_{1}-k_{\mathrm{r}} x_{2}}{1+\frac{x_{1}}{K_{X_{1}}}+\frac{x_{2}}{K_{X_{2}}}}\right)=n_{\mathrm{M}}\left(\frac{k_{\mathrm{f}} x_{1}-k_{\mathrm{r}} x_{2}}{1+\frac{x_{1}}{K_{X_{1}}}+\frac{x_{2}}{K_{X_{2}}}}\right)=\frac{f_{\mathrm{f}} x_{1}-f_{\mathrm{r}} x_{2}}{1+\frac{x_{1}}{K_{X_{1}}}+\frac{x_{2}}{K_{X_{2}}}},
$$

where $m=n_{\mathrm{M}} / A$ is the transporter or enzyme concentration in moldm${ }^{-2}, k_{\mathrm{f}}$ and $k_{\mathrm{r}}$ are the specificity constants in $\mathrm{M}^{-1} \mathrm{~s}^{-1}$, and $K_{X_{1}}$ and $K_{X_{2}}$ are the half-saturation constants for the dissociation of $X$ from the left compartment and right compartment binding sites on the transporter or enzyme. As in eqn. 27, the rate factors are products of the number of transporter or enzyme molecules and rate constants.

\section{A unified view of rate equations for multi-compartment kinetic modelling}

The above analysis revealed that the rate equations for changes in the mole amount of reactants or products per time can be written in a form that is identical to a canonical concentration-change rate law, except that the rate constants are replaced by rate factors. These rate laws differ only in the way the rate factors decompose into

$$
\text { compartment size } \times \text { rate constant, }
$$

where 'compartment size' is a volume, area or length. In the case of intra-compartmental catalysis or mediated inter-compartmental transfer, 'compartment size' is replaced by the number of enzymes, mediators or pores. Both forward and reverse rate factors of course contain the same compartment size. A rate factor is in $\mathrm{dm}^{3} \mathrm{~s}^{-1}$ (if multiplied by one concentration), $\mathrm{dm}^{3} \mathrm{M}^{-1} \mathrm{~s}^{-1}$ (if multiplied by two concentrations), $\mathrm{dm}^{3} \mathrm{M}^{-2} \mathrm{~s}^{-1}$ (if multiplied by three concentrations), etc. 
For the example reaction $X+Y \rightleftharpoons Z$ in Fig. 8 we have the equivalent rate law forms

$$
v=\left(f_{\mathrm{f}} x y-f_{\mathrm{r}} z\right) / D=f_{\mathrm{f}}\left(x y-\frac{z}{K_{\mathrm{eq}}}\right) / D=f_{\mathrm{f}} \cdot x y\left(1-\frac{\Gamma}{K_{\mathrm{eq}}}\right) / D,
$$

where $\Gamma=z / x y$ is the mass-action ratio and $K_{\mathrm{eq}}=f_{\mathrm{f}} / f_{\mathrm{r}}$ is the equilibrium constant (the compartment size always cancels from this ratio to leave the standard definition $K_{\mathrm{eq}}=k_{\mathrm{f}} / k_{\mathrm{r}}$ ). The denominator $D$ is either 1 (for a simple mass-action reaction) or, for a saturable, catalysed reaction, a binding isotherm such as

$$
D=1+\frac{x}{K_{X}}+\frac{y}{K_{Y}}+\frac{z}{K_{Z}}+\frac{x}{K_{X}} \frac{y}{K_{Y}} .
$$

Table 2 shows how the rate factors for the four compartmental configurations in Fig. 8 decompose into compartment size and rate constants.
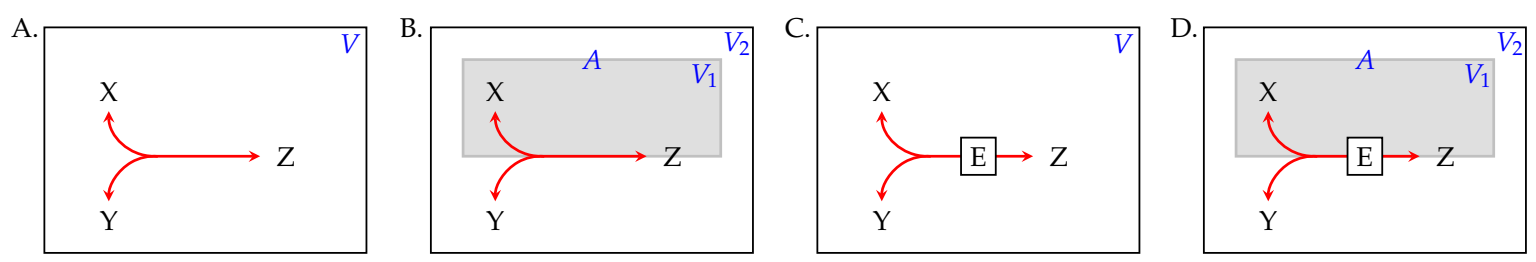

Fig. 8 A bi-uni reaction that occurs throughout a single compartment with volume $V(\mathrm{~A}, \mathrm{C})$ or is confined to the 2-dimensional interface compartment with area $A$ between two 3-dimensional compartments with volumes $V_{1}$ and $V_{2}(\mathrm{~B}, \mathrm{D})$. The reaction is either uncatalysed (A, B) or enzyme-catalysed (C, D).

\section{Rates of processes at compartment boundaries are never directly proportional to a volume}

The literature abounds with examples of models in which rate equations for reactions or transfers that occur at compartment boundaries or interfaces between compartments are scaled in some way or the other with compartment volume(s). The following explains why this is always wrong.

Consider the unidirectional reaction in Fig. 9A. The classical concentration-change first-order rate law, familiar to anyone with a chemistry/biochemistry background, is

$$
-\frac{d x}{d t}=\frac{d y}{d t}=k x
$$

To derive an amount-change rate law the seemingly obvious procedure is to multiply with the volume $V_{1}$ :

$$
-\frac{d n_{X}}{d t}=\frac{d n_{Y}}{d t}=V_{1} \cdot k x
$$


Table 2 Amount-change rate equations for the compartmental configuration in Fig. 8 and the decomposition of their rate factors. Concentrations are explicitly written as mole/compartment size to indicate with respect to which compartment the concentrations are defined. The symbol $\mathrm{M}$ signifies $\mathrm{mol} \mathrm{dm}^{-3}$.

\begin{tabular}{|c|c|l|l|l|l|}
\hline \multirow{2}{*}{ Fig. 8} & \multicolumn{2}{|c|}{$f_{\mathrm{f}}$} & \multicolumn{2}{|c|}{$f_{\mathrm{r}}$} \\
\cline { 2 - 6 } & $v$ & size & \multicolumn{1}{|c|}{$k_{\mathrm{f}}$} & size & \multicolumn{1}{c|}{$k_{\mathrm{r}}$} \\
\hline A. & $f_{\mathrm{f}} \frac{n_{\mathrm{X}}}{V} \frac{n_{\mathrm{Y}}}{V}-f_{\mathrm{r}} \frac{n_{\mathrm{Z}}}{V}$ & $V\left(\mathrm{dm}^{3}\right)$ & $\mathrm{M}^{-1} \mathrm{~s}^{-1}$ & $V\left(\mathrm{dm}^{3}\right)$ & $\mathrm{s}^{-1}$ \\
B. & $f_{\mathrm{f}} \frac{n_{\mathrm{X}}}{V_{1}} \frac{n_{\mathrm{Y}}}{V_{2}}-f_{\mathrm{r}} \frac{n_{\mathrm{Z}}}{A}$ & $A\left(\mathrm{dm}^{2}\right)$ & $\mathrm{dm} \mathrm{M}^{-1} \mathrm{~s}^{-1}$ & $A\left(\mathrm{dm}^{2}\right)$ & $\mathrm{dm} \mathrm{s}^{-1}$ \\
C. & $\left(f_{\mathrm{f}} \frac{n_{\mathrm{X}}}{V} \frac{n_{\mathrm{Y}}}{V}-f_{\mathrm{r}} \frac{n_{\mathrm{Z}}}{V}\right) / D$ & $V e=n_{\mathrm{E}}(\mathrm{mol})^{\mathrm{a}}$ & $\mathrm{M}^{-2} \mathrm{~s}^{-1}$ & $V e=n_{\mathrm{E}}(\mathrm{mol})^{\mathrm{a}}$ & $\mathrm{M}^{-1} \mathrm{~s}^{-1}$ \\
D. & $\left(f_{\mathrm{f}} \frac{n_{\mathrm{X}}}{V_{1}} \frac{n_{\mathrm{Y}}}{V_{2}}-f_{\mathrm{r}} \frac{n_{\mathrm{Z}}}{A}\right) / D$ & $A e=n_{\mathrm{E}}(\mathrm{mol})^{\mathrm{b}}$ & $\mathrm{M}^{-2} \mathrm{~s}^{-1}$ & $A e=n_{\mathrm{E}}(\mathrm{mol})^{\mathrm{b}}$ & $\mathrm{M}^{-1} \mathrm{~s}^{-1}$ \\
\hline
\end{tabular}

a Enzyme concentration $e$ in $\operatorname{mol~} \mathrm{dm}^{-3} ;{ }^{\mathrm{b}} e$ in $\mathrm{mol} \mathrm{dm}{ }^{-2}$.

A.

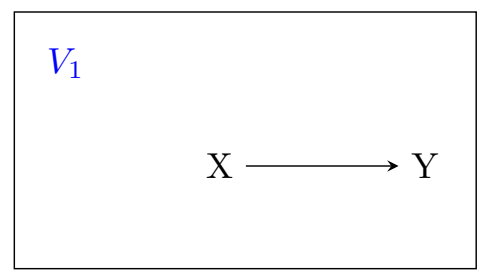

B.

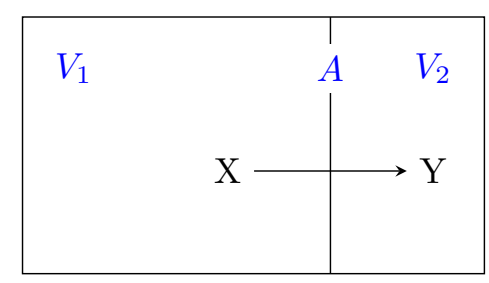

C.

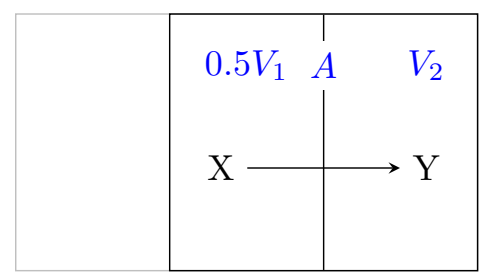

Fig. 9 Three compartmental configurations. In B and C the reaction is considered to be either a chemical reaction or a transfer process, in which case $Y$ distinguishes $X$ in compartment 2 from $X$ in compartment 1.

This approach, however, only works for situations such as Fig. 9A where all reactants and products occur in the same volume compartment and where the reaction events actually occur within that compartment; it can therefore not be applied to Figs. 9B and $C$ where the transfer events occur on the boundary between the two compartments. ${ }^{5}$ That volume scaling is wrong in all cases where the reaction or transfer takes place at the boundary of a compartment or in the interface between compartments is serious, since it forms the basis for an erroneous general procedure for handling rate laws, irrespective of whether they are for reactions in a compartment, at the boundary of a compartment, or at the interface between compartments.

The reason for the error is simple: for Fig. 9A it is eqn. 35 that is the fundamental

\footnotetext{
${ }^{5}$ Nor, for that matter, Fig. $5 \mathrm{C}$ where the reactants and products all occur in the same compartment but the reaction events take place on the compartment boundary.
} 
rate equation conforming to $v=d \xi / d t$; eqn. 34 should be derived from it by dividing with $V_{1}$, and not, as was done above, the other way round. As established in the foregoing, the correct starting point for Figs. 9B and $\mathrm{C}$ is the amount-change rate equation

$$
v=\frac{d \xi}{d t}=-\frac{d n_{X}}{d t}=\frac{d n_{Y}}{d t}=A \cdot k x
$$

from which, by dividing with the appropriate volume, the correct concentrationchange rate equations are derived as

$$
-\frac{d x}{d t}=\frac{A}{V_{1}} \cdot k x \quad \text { and } \quad \frac{d y}{d t}=\frac{A}{V_{2}} \cdot k x
$$

There is also a decisive thermodynamic reason why volume scaling in Figs. 9B and C is incorrect. All chemical reactions and transport processes are in principle reversible, even if their equilibrium constants are very large. Logic dictates that the reasoning that led to eqn. 35 must also apply to the reverse reaction, so that for a bi-directional boundary-crossing reaction $X \rightleftharpoons Y$ the rate would be

$$
v=-\frac{d n_{X}}{d t}=\frac{d n_{Y}}{d t}=V_{1} \cdot k_{\mathrm{f}} x-V_{2} \cdot k_{\mathrm{r}} y
$$

i.e., the forward rate term is multiplied with the volume in which the reactant(s) occur, while the reverse rate term is multiplied with the volume in which the product(s) occur. However, this rate equation is thermodynamically inconsistent. At equilibrium $v=0$, which yields

$$
K_{\mathrm{eq}}=\frac{y_{\mathrm{eq}}}{x_{\mathrm{eq}}}=\frac{k_{\mathrm{f}}}{k_{\mathrm{r}}} \cdot \frac{V_{1}}{V_{2}}
$$

Because the equilibrium constant expression contains a volume ratio, it does not reduce to the required ratio of the forward and reverse rate constants (except when $V_{1}=V_{2}$ ). This means that changing the volume of one compartment selectively alters the value of the equilibrium constant, which of course violates a basic principle of chemical thermodynamics.

Here is another way of looking at it: Consider $X \rightleftharpoons Y$ to represent a simple bidirectional diffusion of a substance $X$ over a membrane between two compartments with unequal volumes $V_{1}$ and $V_{2}$ ( $y$ being the concentration of $X$ in compartment with volume $V_{2}$ ). Since here $K_{\mathrm{eq}}=k_{\mathrm{f}} / k_{\mathrm{r}}=1$, the equilibrium concentrations of $\mathrm{X}$ and $\mathrm{Y}$ must be equal. Set eqn. 38 to zero. Are $x$ and $y$ equal? No. Alternatively, set $x=y$ and calculate the rate. Is it zero, as it should be at equilibrium? No.

From eqn. 38 the following concentration-change rate equations, similarly erroneous, can be generated:

$$
-\frac{d x}{d t}=k_{\mathrm{f}} x-k_{\mathrm{r}} y \cdot \frac{V_{2}}{V_{1}} \quad \text { and } \quad \frac{d y}{d t}=\frac{V_{1}}{V_{2}} \cdot k_{\mathrm{f}} x-k_{\mathrm{r}} y
$$


Many published models use such rate equations and seem to get away with it because the compartment volumes are fixed. ${ }^{6}$ Nevertheless, these models are fundamentally flawed. The correct (and thermodynamically consistent) form of the concentrationchange rate laws is

$$
-\frac{d x}{d t}=\frac{A}{V_{1}}\left(k_{\mathrm{f}} x-k_{\mathrm{r}} y\right) \text { and } \frac{d y}{d t}=\frac{A}{V_{2}}\left(k_{\mathrm{f}} x-k_{\mathrm{r}} y\right)
$$

or, if the compartment geometry and therefore the boundary area is unknown,

$$
-\frac{d x}{d t}=\frac{1}{V_{1}}\left(f_{\mathrm{f}} x-f_{\mathrm{r}} y\right) \text { and } \frac{d y}{d t}=\frac{1}{V_{2}}\left(f_{\mathrm{f}} x-f_{\mathrm{r}} y\right),
$$

in which both $k_{\mathrm{f}}$ and $k_{\mathrm{r}}$ are scaled by the same (but unquantified) factor $A$, so that the rate factors $f_{\mathrm{f}}$ and $f_{\mathrm{r}}$ would have to be determined by fitting to the experimental data for a particular fixed compartmental geometry.

If the boundary area is unknown but the number of mediateors or pores $n_{\mathrm{M}}$ are, then

$$
-\frac{d x}{d t}=\frac{n_{\mathrm{M}}}{V_{1}}\left(k_{\mathrm{f}} x-k_{\mathrm{r}} y\right) \quad \text { and } \quad \frac{d y}{d t}=\frac{n_{\mathrm{M}}}{V_{2}}\left(k_{\mathrm{f}} x-k_{\mathrm{r}} y\right) .
$$

I have also encountered instances where the full reversible rate equation is multiplied with the volume of the compartment in which the reactants occur

$$
v=-\frac{d n_{\mathrm{X}}}{d t}=\frac{d n_{\mathrm{Y}}}{d t}=V_{1}\left(k_{\mathrm{f}} x-k_{\mathrm{r}} y\right)
$$

which seems to circumvent the thermodynamic problem, since at $v=0$ the correct expression for the equilibrium constant obtains. However, as expressed here the amount-change rate depends explicitly on $V_{1}$, but not on $V_{2}$. This is patently nonsense. If both the forward and reverse rate terms are to be affected by volume changes, then the effect cannot be limited to one volume only. This also shows up the arbitrariness of multiplying the rate law with $V_{1}$. Why not multiply with $V_{2}$ ? This would yield

$$
v=-\frac{d n_{\mathrm{X}}}{d t}=\frac{d n_{\mathrm{Y}}}{d t}=V_{2}\left(k_{\mathrm{f}} x-k_{\mathrm{r}} y\right)
$$

The two forms of the rate law must yield the same value, which again is the case only when $V_{1}=V_{2}$, which is clearly not the intention.

Lastly, we have already encountered in Section 5 an operational reason why volume scaling in Figs. 9B and $\mathrm{C}$ is incorrect. In Fig. 9C $V_{1}$ halves in size while the interface area remains the same. Let $V_{1}$ in both cases have the same concentration of $X$, and take into account that the transfer takes place only at the interface between the two compartments. What eqn. 35 tells us is that for the unidirectional reaction the

\footnotetext{
${ }^{6}$ I invite you to trawl through the EBI BioModels database [5], where you will find a number of models that fit this bill, e.g., [6]. You will also find models that handle compartmentation correctly, e.g., [7].
} 
amount of $X$ that gets transferred per time unit in Fig. 9C will be half of that in Fig. 9B, despite the size of the interface where the reaction events occur being the same. This is clearly impossible, because the concentration of $X$ at the interface (to which the rate is proportional) is the same in both instances. The reaction rate is proportional to the interface area $A$, not to the volume $V_{1}$ (recall lesson 1 in Section 4).

I hope I have now finally laid to rest the erroneous procedure of multiplying with a compartment volume the rate law for a process that occurs at a compartment boundary or at the interface between compartments.

\section{Incorporating rate equations in ODEs}

The systems of ordinary differential equations (ODEs) that describe the dynamics of compartmentalised reaction networks can be cast in either amount per time or concentration per time changes, and we have to consider both these possibilities for models in which the compartment sizes are fixed and those in which they change.

Consider a simple system with two coupled reactions that occur either in a single compartment or spread out over three compartments (Fig. 10). Amount-change rates $v_{1}$ and $v_{2}$ can be those of chemical reactions that occur in the single compartment of Fig. 10A, or occur on the boundaries between the compartments of Fig. 10B (where $X, Y$, and $Z$ are difference substances); they can also be the rates of transfer events between the compartments (where $X, Y$, and $Z$ are the same substance).
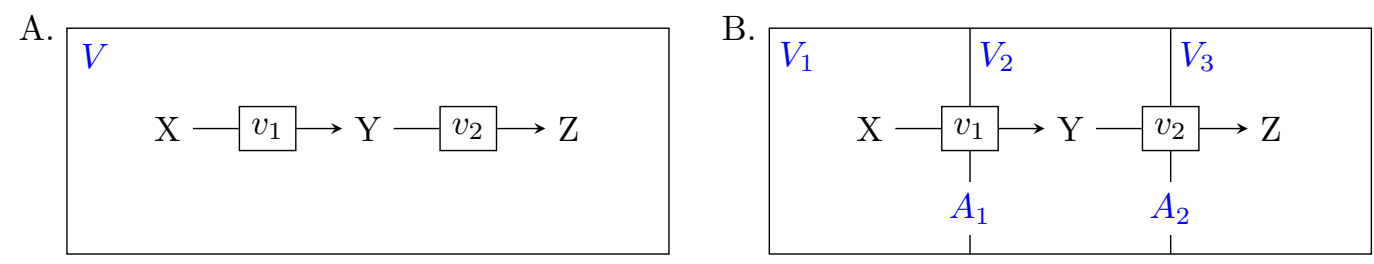

Fig. 10 A system of two coupled reactions in A. a single compartment and B. spread over three compartments.

Fig. 10A may seem trivial, since if the compartment volume $V$ is fixed one would usually model with canonical concentration-change rate laws and substance concentrations, and not even consider the volume. Nevertheless, it serves as a benchmark for comparison with the compartmental configuration in Fig. 10B. I also need to consider the situation where the volumes and boundary areas are not fixed, but vary in time, not only for Fig. 10B but also for Fig. 10A.

The amount-change rates $v_{1}$ and $v_{2}$ must be calculated from appropriate rate equations. Our stated aims were to (1) use amount-change rate equations based on the definition of reaction rate in terms of reaction events per time, (2) derive these rate equations from the canonical concentration-change rate laws, and (3) formulate them in such a way that they need to be specified only once for each reaction. 
The canonical concentration-change rate laws for the reactions in Fig. 10 are

$$
\begin{aligned}
& \mathcal{R}_{1}=e_{1}\left(k_{1 \mathrm{f}} x-k_{1 \mathrm{r}} y\right) / D \\
& \mathcal{R}_{2}=e_{2}\left(k_{2 \mathrm{f}} y-k_{2 \mathrm{r}} z\right) / D,
\end{aligned}
$$

where for uncatalysed, mass-action reactions $e_{1}=e_{2}=1$ and $D=1$, and for enzymecatalysed reactions $e_{1}$ and $e_{2}$ are variables and

$$
D=1+\frac{s}{K_{\mathrm{S}}}+\frac{p}{K_{\mathrm{P}}}
$$

for the $(\mathrm{S}, \mathrm{P})$-pairs $(\mathrm{X}, \mathrm{Y})$ and $(\mathrm{Y}, \mathrm{Z})$.

Table 3 lists the different forms of the amount-change rate equations for the uncatalysed and the enzyme-catalysed reactions using either concentrations or mole amounts. Whereas in the single compartment model the reaction events occur in volume $V$, the reaction or transfer events in the multi-compartment model are localised

\begin{tabular}{|c|c|c|c|c|}
\hline \multirow[b]{2}{*}{ Fig. 10A } & \multicolumn{2}{|l|}{$v_{1}$} & \multicolumn{2}{|l|}{$v_{2}$} \\
\hline & $\begin{array}{l}V\left(k_{1 \mathrm{f}} x-k_{1 \mathrm{r}} y\right) \\
V\left(k_{1 \mathrm{f}} \frac{n_{\mathrm{X}}}{V}-k_{1 \mathrm{r}} \frac{n_{\mathrm{Y}}}{V}\right) \\
n_{\mathrm{E}_{1}}\left(k_{1 \mathrm{f}} x-k_{1 \mathrm{r}} y\right) / D \\
n_{\mathrm{E}_{1}}\left(k_{1 \mathrm{f}} \frac{n_{\mathrm{X}}}{V}-k_{1 \mathrm{r}} \frac{n_{\mathrm{Y}}}{V}\right) / D\end{array}$ & $\begin{array}{l}V \mathcal{R}_{1} \\
V \mathcal{R}_{1} \\
V \mathcal{R}_{1} \\
V \mathcal{R}_{1}\end{array}$ & $\begin{array}{l}V\left(k_{2 \mathrm{f}} y-k_{2 \mathrm{r}} z\right) \\
V\left(k_{2 \mathrm{f}} \frac{n_{\mathrm{Y}}}{V}-k_{2 \mathrm{r}} \frac{n_{\mathrm{Z}}}{V}\right) \\
n_{\mathrm{E}_{2}}\left(k_{2 \mathrm{f}} y-k_{2 \mathrm{r}} z\right) / D \\
n_{\mathrm{E}_{2}}\left(k_{2 \mathrm{f}} \frac{n_{\mathrm{Y}}}{V}-k_{2 \mathrm{r}} \frac{n_{\mathrm{Z}}}{V}\right) / D\end{array}$ & $\begin{array}{l}V \mathcal{R}_{2} \\
V \mathcal{R}_{2} \\
V \mathcal{R}_{2} \\
V \mathcal{R}_{2}\end{array}$ \\
\hline Fig. 10B & $\begin{array}{l}A_{1}\left(k_{1 \mathrm{f}} x-k_{1 \mathrm{r}} y\right) \\
A_{1}\left(k_{1 \mathrm{f}} \frac{n_{\mathrm{X}}}{V_{1}}-k_{1 \mathrm{r}} \frac{n_{\mathrm{Y}}}{V_{2}}\right) \\
n_{\mathrm{E}_{1}}\left(k_{1 \mathrm{f}} x-k_{1 \mathrm{r}} y\right) / D \\
n_{\mathrm{E}_{1}}\left(k_{1 \mathrm{f}} \frac{n_{\mathrm{X}}}{V_{1}}-k_{1 \mathrm{r}} \frac{n_{\mathrm{Y}}}{V_{2}}\right) / D\end{array}$ & $\begin{array}{l}A_{1} \mathcal{R}_{1} \\
A_{1} \mathcal{R}_{1} \\
A_{1} \mathcal{R}_{1} \\
A_{1} \mathcal{R}_{1}\end{array}$ & $\begin{array}{l}A_{2}\left(k_{2 \mathrm{f}} y-k_{2 \mathrm{r}} z\right) \\
A_{2}\left(k_{2 \mathrm{f}} \frac{n_{\mathrm{Y}}}{V_{2}}-k_{2 \mathrm{r}} \frac{n_{\mathrm{Z}}}{V_{3}}\right) \\
n_{\mathrm{E}_{2}}\left(k_{2 \mathrm{f}} y-k_{2 \mathrm{r}} z\right) / D \\
n_{\mathrm{E}_{2}}\left(k_{2 \mathrm{f}} \frac{n_{\mathrm{Y}}}{V_{2}}-k_{2 \mathrm{r}} \frac{n_{\mathrm{Z}}}{V_{3}}\right) / D\end{array}$ & $\begin{array}{l}A_{2} \mathcal{R}_{2} \\
A_{2} \mathcal{R}_{2} \\
A_{2} \mathcal{R}_{2} \\
A_{2} \mathcal{R}_{2}\end{array}$ \\
\hline
\end{tabular}
on the two boundaries with areas $A_{1}$ and $A_{2}$ that separate the compartments.

Table 3 Amount-change rate equations for the reactions in Fig. 10.

As will become abundantly clear, ODEs for changes in the mole amounts of substances are the simplest, and therefore the preferred formulation. Since in the amountchange ODEs the system variables are mole amounts, is is often useful to use those rate equations in Table 3 that are also formulated in terms of mole amounts. It is of course always possible (often even preferable) to use the amount-change rate equations formulated in terms of concentrations, but then each concentration needs to be 
recalculated from the mole amount and the compartment size after every iteration of the ODE-solver.

Concentration-change ODEs are derived from the amount-change ODEs as follows: For any substance $\mathrm{X}$ with concentration $x$ in volume $V$

$$
\frac{d n_{\mathrm{X}}}{d t}=\frac{d(V x)}{d t}=V \frac{d x}{d t}+x \frac{d V}{d t}
$$

Re-arranging and dividing by $V$ gives the ODE for the concentration $x$ :

$$
\frac{d x}{d t}=\frac{1}{V} \frac{d n_{\mathrm{X}}}{d t}-\frac{x}{V} \frac{d V}{d t}
$$

where $\frac{1}{V} \frac{d n_{\mathrm{X}}}{d t}$ is the rate of change in $x$ due to $\mathrm{X}$ being produced, consumed or transferred, and $\frac{x}{V} \frac{d V}{d t}$ is the rate of change in $x$ due to a change in volume (sometimes, rather misleadingly, referred to as the "flux to expansion").

If $\mathrm{X}$ is confined to a two-dimensional boundary with area $A$ then

$$
\frac{d x}{d t}=\frac{1}{A} \frac{d n_{\mathrm{X}}}{d t}-\frac{x}{A} \frac{d A}{d t}
$$

For the moment we regard the volumes and boundary areas as fixed, so that $d V / d t=$ $d A / d t=0$ and

$$
\frac{d x}{d t}=\frac{1}{V} \frac{d n_{\mathrm{X}}}{d t} \quad \text { or } \quad \frac{d x}{d t}=\frac{1}{A} \frac{d n_{\mathrm{X}}}{d t} .
$$

In concentration-change ODEs, the concentration-based rate equations in Table 3 must be used.

\subsection{ODEs for fixed-size single-compartment systems}

For the single-compartment configuration in Fig. 10A the amount-change ODEs are:

$$
\begin{aligned}
& \frac{d n_{\mathrm{X}}}{d t}=-v_{1} \quad=-V \mathcal{R}_{1} \\
& \frac{d n_{\mathrm{Y}}}{d t}=v_{1}-v_{2}=V \mathcal{R}_{1}-V \mathcal{R}_{2} \\
& \frac{d n_{\mathrm{Z}}}{d t}=v_{2}=V \mathcal{R}_{2}
\end{aligned}
$$

As a matrix equation that incorporates the stoichiometry matrix the amount-change ODEs are written as

$$
\frac{d}{d t}\left[\begin{array}{l}
n_{\mathrm{X}} \\
n_{\mathrm{Y}} \\
n_{\mathrm{Z}}
\end{array}\right]=\left[\begin{array}{cc}
-1 & 0 \\
1 & -1 \\
0 & 1
\end{array}\right]\left[\begin{array}{l}
v_{1} \\
v_{2}
\end{array}\right]
$$


or, with canonical rate equations,

$$
\frac{d}{d t}\left[\begin{array}{l}
n_{\mathrm{X}} \\
n_{\mathrm{Y}} \\
n_{\mathrm{Z}}
\end{array}\right]=\left[\begin{array}{cc}
-1 & 0 \\
1 & -1 \\
0 & 1
\end{array}\right]\left[\begin{array}{ll}
V & 0 \\
0 & V
\end{array}\right]\left[\begin{array}{l}
\mathcal{R}_{1} \\
\mathcal{R}_{2}
\end{array}\right]
$$

Symbolically, the amount-change ODE matrix equation is

$$
\frac{d \mathbf{n}}{d t}=\mathbf{N v}=\mathbf{N} \cdot \operatorname{diag} C_{\mathrm{r}} \cdot \mathcal{R}
$$

with $\mathbf{n}$ a vector of mole amounts, $\mathbf{N}$ the stoichiometry matrix, $\operatorname{diag} C_{\mathrm{r}}$ a diagonal matrix of the compartment volume $V, \mathbf{v}$ the vector of amount-change rates, and $\mathcal{R}$ the vector of canonical concentration-change rate laws (for a full description of the kinetic model in terms of symbolic matrix algebra, see Appendix A).

Because $\mathrm{X}, \mathrm{Y}$ and $\mathrm{Z}$ are all here regarded as variable, the three ODEs sum to zero,

$$
\frac{d n_{X}}{d t}+\frac{d n_{Y}}{d t}+\frac{d n_{Z}}{d t}=0
$$

which means that they are linearly dependent, so that

$$
n_{\mathrm{X}}+n_{\mathrm{y}}+n_{\mathrm{Z}}=\text { constant. }
$$

To obtain a set of independent equations, one of the ODEs must be replaced by this so-called moiety-conservation equation. Moiety conservation in the context of compartmental modelling will be discussed in Section 12 .

Dividing the amount-change ODEs by volume $V$ yields the concentration-change ODEs for Fig. 10A:

$$
\frac{d}{d t}\left[\begin{array}{l}
x \\
y \\
x
\end{array}\right]=\left[\begin{array}{ccc}
\frac{1}{V} & 0 & 0 \\
0 & \frac{1}{V} & 0 \\
0 & 0 & \frac{1}{V}
\end{array}\right]\left[\begin{array}{cc}
-1 & 0 \\
1 & -1 \\
0 & 1
\end{array}\right]\left[\begin{array}{l}
v_{1} \\
v_{2}
\end{array}\right]
$$

or, with canonical rate equations,

$$
\frac{d}{d t}\left[\begin{array}{l}
x \\
y \\
x
\end{array}\right]=\left[\begin{array}{ccc}
\frac{1}{V} & 0 & 0 \\
0 & \frac{1}{V} & 0 \\
0 & 0 & \frac{1}{V}
\end{array}\right]\left[\begin{array}{cc}
-1 & 0 \\
1 & -1 \\
0 & 1
\end{array}\right]\left[\begin{array}{ll}
V & 0 \\
0 & V
\end{array}\right]\left[\begin{array}{l}
\mathcal{R}_{1} \\
\mathcal{R}_{2}
\end{array}\right]
$$


which, as required for a single compartment with fixed volume, expands to

$$
\begin{array}{ll}
\frac{d x}{d t}=-\frac{1}{V} v_{1} & =-\mathcal{R}_{1} \\
\frac{d y}{d t}=\frac{1}{V} v_{1}-\frac{1}{V} v_{2} & =\mathcal{R}_{1}-\mathcal{R}_{2} \\
\frac{d z}{d t}=\frac{1}{V} v_{2} & =\mathcal{R}_{2} .
\end{array}
$$

This is the seemingly trivial situation referred to above, where one models with canonical concentration-change rate laws and substance concentrations without considering the volume.

Note that for the single-compartment model in Fig. 10A the three concentrationchange ODEs still sum to zero, which means that there is moiety conservation with

$$
x+y+z=\text { constant. }
$$

Symbolically, the concentration-change ODE matrix equation for the single compartment with fixed volume is

$$
\frac{d \mathbf{s}}{d t}=\left(\operatorname{diag} C_{\mathrm{s}}\right)^{-1} \cdot \mathbf{N} \cdot \operatorname{diag} C_{\mathrm{r}} \cdot \mathcal{R}=\mathbf{N} \mathcal{R}
$$

with $\mathbf{s}$ a vector of concentrations, and $\operatorname{diag} C_{\mathrm{s}}$ a diagonal matrix of the compartment sizes associated with the substances, here the volume $V$.

For fixed-size single compartment systems the amount-change ODEs $\left(d \mathbf{n}_{\mathrm{X}} / d t=\right.$ $\mathbf{N v}$ ) and concentration-change ODEs $(d \mathbf{s} / d t=\mathbf{N} \mathcal{R})$ have the same form, and differ only in terms of the rate equations used: amount-change rate equations for the former and canonical concentration-change rate equations for the latter.

\subsection{ODEs for fixed-size multi-compartment systems}

For Fig. 10B the amount-change ODEs with rate equations are:

$$
\begin{aligned}
& \frac{d n_{\mathrm{X}}}{d t}=-v_{1} \quad=-A_{1} \mathcal{R}_{1} \\
& \frac{d n_{\mathrm{Y}}}{d t}=v_{1}-v_{2}=A_{1} \mathcal{R}_{1}-A_{2} \mathcal{R}_{2} \\
& \frac{d n_{\mathrm{Z}}}{d t}=v_{2}=A_{2} \mathcal{R}_{2}
\end{aligned}
$$

The matrix equation for the amount-change ODEs has exactly the same form as that for the single-compartment system:

$$
\frac{d}{d t}\left[\begin{array}{l}
n_{\mathrm{X}} \\
n_{\mathrm{Y}} \\
n_{\mathrm{Z}}
\end{array}\right]=\left[\begin{array}{cc}
-1 & 0 \\
1 & -1 \\
0 & 1
\end{array}\right]\left[\begin{array}{l}
v_{1} \\
v_{2}
\end{array}\right] .
$$


The difference lies in the rate equations, since the reaction events are now associated with the membranes that separate the compartments. With canonical concentrationchange rate laws the matrix equation is

$$
\frac{d}{d t}\left[\begin{array}{l}
n_{\mathrm{X}} \\
n_{\mathrm{Y}} \\
n_{\mathrm{Z}}
\end{array}\right]=\left[\begin{array}{cc}
-1 & 0 \\
1 & -1 \\
0 & 1
\end{array}\right]\left[\begin{array}{cc}
A_{1} & 0 \\
0 & A_{2}
\end{array}\right]\left[\begin{array}{l}
\mathcal{R}_{1} \\
\mathcal{R}_{2}
\end{array}\right] .
$$

The three ODEs still sum to zero so that

$$
n_{\mathrm{X}}+n_{\mathrm{y}}+n_{\mathrm{Z}}=\text { constant. }
$$

In symbolic terms the amount-change ODE matrix equation is, as for the singlecompartment configuration,

$$
\frac{d \mathbf{n}}{d t}=\mathbf{N} \mathbf{v}=\mathbf{N} \cdot \operatorname{diag} C_{\mathbf{r}} \cdot \mathcal{R},
$$

but now with $\operatorname{diag} C_{\mathrm{r}}$ containing membrane areas instead of the compartment volume.

Dividing each amount-change ODE by its corresponding volume yields the concentrationchange ODEs:

$$
\frac{d}{d t}\left[\begin{array}{l}
x \\
y \\
x
\end{array}\right]=\left[\begin{array}{ccc}
\frac{1}{V_{1}} & 0 & 0 \\
0 & \frac{1}{V_{2}} & 0 \\
0 & 0 & \frac{1}{V_{3}}
\end{array}\right]\left[\begin{array}{cc}
-1 & 0 \\
1 & -1 \\
0 & 1
\end{array}\right]\left[\begin{array}{c}
v_{1} \\
v_{2}
\end{array}\right]
$$

or, with canonical concentration-change rate laws,

$$
\frac{d}{d t}\left[\begin{array}{l}
x \\
y \\
x
\end{array}\right]=\left[\begin{array}{ccc}
\frac{1}{V_{1}} & 0 & 0 \\
0 & \frac{1}{V_{2}} & 0 \\
0 & 0 & \frac{1}{V_{3}}
\end{array}\right]\left[\begin{array}{cc}
-1 & 0 \\
1 & -1 \\
0 & 1
\end{array}\right]\left[\begin{array}{cc}
A_{1} & 0 \\
0 & A_{2}
\end{array}\right]\left[\begin{array}{l}
\mathcal{R}_{1} \\
\mathcal{R}_{2}
\end{array}\right],
$$

which expands to

$$
\begin{aligned}
& \frac{d x}{d t}=-\frac{1}{V_{1}} v_{1} \quad=-\frac{1}{V_{1}} A_{1} \mathcal{R}_{1} \\
& \frac{d y}{d t}=\frac{1}{V_{2}} v_{1}-\frac{1}{V_{2}} v_{2}=\frac{1}{V_{2}} A_{1} \mathcal{R}_{1}-\frac{1}{V_{2}} A_{2} \mathcal{R}_{2} \\
& \frac{d z}{d t}=\frac{1}{V_{3}} v_{2} \quad=\frac{1}{V_{3}} A_{2} \mathcal{R}_{2}
\end{aligned}
$$


In contrast to the concentration-change ODEs in the single-compartment model, the concentration-change ODEs in the multi-compartment model do not sum to zero, which means that although the sum of mole amounts of $X, Y$, and $Z$ is constant, the sum of their concentrations is not.

As for the single-compartment system, the concentration-change ODE matrix equation is

$$
\frac{d \mathbf{s}}{d t}=\left(\operatorname{diag} C_{\mathrm{s}}\right)^{-1} \cdot \mathbf{N v}=\left(\operatorname{diag} C_{\mathrm{s}}\right)^{-1} \cdot \mathbf{N} \cdot \operatorname{diag} C_{\mathrm{r}} \cdot \mathcal{R},
$$

but here the matrix equation does not simplify to $d \mathbf{s} / d t=\mathbf{N} \mathcal{R}$.

In summary, with regard to the aim of specifying rate equations only once, the above analysis showed that, depending on the situation, this is always possible using either $\mathcal{R}, V \mathcal{R}$, or $A \mathcal{R}$.

\subsection{ODEs that use amount-change rate equations with rate factors}

Situations may arise where the compartmental geometry in Fig. 10B is unknown, i.e., while it is known that the system contains reactions or transfers that take place across compartmental boundaries, the sizes of the boundary areas are unknown. At least for those reactions one is forced to use amount-change rate equations with rate factors such as those in Table 4, but it is of course possible to use these rate equations for the reactions in Fig. 10A as well. Nevertheless, even though the compartment sizes may be unknown, it is useful to understand the internal structure of the rate factors: for the uncatalysed reactions in Fig. 10A the rate factors are $f_{\mathrm{f}}=V k_{\mathrm{f}}$ and $f_{\mathrm{r}}=V k_{\mathrm{r}}$ with $k_{\mathrm{f}}$ and $k_{\mathrm{r}}$ in s ${ }^{-1}$, and for Fig. 10B $f_{\mathrm{f}}=A k_{\mathrm{f}}$ and $f_{\mathrm{r}}=A k_{\mathrm{r}}$ with $k_{\mathrm{f}}$ and $k_{\mathrm{r}}$ in dm s${ }^{-1}$. For enzyme-catalysed reactions in both Fig. 10A and B the rate factors are $f_{\mathrm{f}}=n_{\mathrm{E}} k_{\mathrm{f}}$ and $f_{\mathrm{r}}=n_{\mathrm{E}} k_{\mathrm{r}}$ with $k_{\mathrm{f}}$ and $k_{\mathrm{r}}$ in $\mathrm{M}^{-1} \mathrm{~s}^{-1}$.

The rate equations in Table 4 are used with the amount-change ODEs for both Fig. 10A and B (eqn. 56), and with the concentration-change ODEs for Fig. 10A (eqn. 61) and Fig. 10B (eqn. 75). Note again that the use of rate equations with rate factors precludes models in which the compartment sizes change with time.

Finally, another possible situation is that, although the compartmental geometry is unknown, the number of mediators or pores in the interfaces are known, so that one can use the rate equations in Table 3 that are scaled with $n_{\mathrm{E}_{1}}$ and $n_{\mathrm{E}_{2}}$.

\subsection{ODEs for variable-size compartmentalised systems}

By far the simplest strategy for modelling the compartmentalised systems in both Fig. 10A and B in situations where the compartment sizes vary is to use the amount- 
Table 4 Amount-change rate equations formulated in terms of rate factors for the reactions in Fig. 10.

\begin{tabular}{|c|c|c|}
\hline & $v_{1}$ & $v_{2}$ \\
\hline Fig. 10A & $\begin{array}{l}f_{1 \mathrm{f}} x-f_{1 \mathrm{r}} y \\
f_{1 \mathrm{f}} \frac{n_{\mathrm{X}}}{V}-f_{1 \mathrm{r}} \frac{n_{Y}}{V} \\
\left(f_{1 \mathrm{f}} x-f_{1 \mathrm{r}} y\right) / D \\
\left(f_{1 \mathrm{f}} \frac{n_{X}}{V}-f_{1 \mathrm{r}} \frac{n_{Y}}{V}\right) / D\end{array}$ & $\begin{array}{l}f_{2 \mathrm{f}} y-f_{2 \mathrm{r}} z \\
f_{2 \mathrm{f}} \frac{n_{Y}}{V}-f_{2 \mathrm{r}} \frac{n_{\mathrm{Z}}}{V} \\
\left(f_{2 \mathrm{f}} y-f_{2 \mathrm{r}} z\right) / D \\
\left(f_{2 \mathrm{f}} \frac{n_{Y}}{V}-f_{2 \mathrm{r}} \frac{n_{\mathrm{Z}}}{V}\right) / D\end{array}$ \\
\hline Fig. 10B & $\begin{array}{l}f_{1 \mathrm{f}} x-f_{1 \mathrm{r}} y \\
f_{1 \mathrm{f}} \frac{n_{\mathrm{X}}}{V_{1}}-f_{1 \mathrm{r}} \frac{n_{Y}}{V_{2}} \\
\left(f_{1 \mathrm{f}} x-f_{1 \mathrm{r}} y\right) / D \\
\left(f_{1 \mathrm{f}} \frac{n_{\mathrm{X}}}{V_{1}}-f_{1 \mathrm{r}} \frac{n_{Y}}{V_{2}}\right) / D\end{array}$ & $\begin{array}{l}f_{2 \mathrm{f}} y-f_{2 \mathrm{r}} z \\
f_{2 \mathrm{f}} \frac{n_{\mathrm{Y}}}{V_{2}}-f_{2 \mathrm{r}} \frac{n_{\mathrm{Z}}}{V_{3}} \\
\left(f_{2 \mathrm{f}} \mathrm{y}-f_{2 \mathrm{r}} z\right) / D \\
\left(f_{2 \mathrm{f}} \frac{n_{Y}}{V_{2}}-f_{2 \mathrm{r}} \frac{n_{\mathrm{Z}}}{V_{3}}\right) / D\end{array}$ \\
\hline
\end{tabular}

change ODEs, $d \mathbf{n} / d t=\mathbf{N v}$ :

$$
\frac{d}{d t}\left[\begin{array}{l}
n_{\mathrm{X}} \\
n_{\mathrm{Y}} \\
n_{\mathrm{Z}}
\end{array}\right]=\left[\begin{array}{cc}
-1 & 0 \\
1 & -1 \\
0 & 1
\end{array}\right]\left[\begin{array}{l}
v_{1} \\
v_{2}
\end{array}\right]
$$

All that needs to be added are ODEs for the rates of change of compartment sizes such as volumes and areas, whichever are applicable (see the example in Appendix D). If concentration-based rate equations are used, the concentrations must be updated after every iteration of the ODE-solver using the new values of mole amounts and compartment sizes.

If, for some reason, one wishes to use concentration-change ODEs, then for Fig. 10A they are (keeping in mind that $v_{1}=V \mathcal{R}_{1}$ and $v_{2}=V \mathcal{R}_{2}$ )

$$
\begin{aligned}
\frac{d x}{d t}=-\frac{1}{V} v_{1}-\frac{x}{V} \frac{d V}{d t} & =\mathcal{R}_{1}-\frac{x}{V} \frac{d V}{d t} \\
\frac{d y}{d t}=\frac{1}{V} v_{1}-\frac{1}{V} v_{2}-\frac{y}{V} \frac{d V}{d t} & =\mathcal{R}_{1}-\mathcal{R}_{2}-\frac{y}{V} \frac{d V}{d t} \\
\frac{d z}{d t}=\frac{1}{V} v_{2}-\frac{z}{V} \frac{d V}{d t} & =\mathcal{R}_{2}-\frac{z}{V} \frac{d V}{d t}
\end{aligned}
$$


A separate ODE must be supplied for volume $V$.

For Fig. 10B:

$$
\begin{array}{ll}
\frac{d x}{d t}=-\frac{1}{V_{1}} v_{1}-\frac{x}{V_{1}} \frac{d V_{1}}{d t} & =\frac{1}{V_{1}} A_{1} \mathcal{R}_{1}-\frac{x}{V_{1}} \frac{d V_{1}}{d t} \\
\frac{d y}{d t}=\frac{1}{V_{2}} v_{1}-\frac{1}{V_{2}} v_{2}-\frac{y}{V_{2}} \frac{d V_{2}}{d t} & =\frac{1}{V_{2}} A_{1} \mathcal{R}_{1}-\frac{1}{V_{2}} A_{2} \mathcal{R}_{2}-\frac{y}{V_{2}} \frac{d V_{2}}{d t} \\
\frac{d z}{d t}=\frac{1}{V_{3}} v_{2}-\frac{z}{V_{3}} \frac{d V_{3}}{d t} & =\frac{1}{V_{3}} A_{2} \mathcal{R}_{2}-\frac{z}{V_{3}} \frac{d V_{3}}{d t}
\end{array}
$$

Again, where applicable, additional ODEs must be supplied for $V_{1}, V_{2}, V_{3}, A_{1}$, and $A_{2}$.

In matrix form,

$$
\frac{d}{d t}\left[\begin{array}{l}
x \\
y \\
x
\end{array}\right]=\left[\begin{array}{ccc}
\frac{1}{V_{1}} & 0 & 0 \\
0 & \frac{1}{V_{2}} & 0 \\
0 & 0 & \frac{1}{V_{3}}
\end{array}\right]\left(\left[\begin{array}{cc}
-1 & 0 \\
1 & -1 \\
0 & 1
\end{array}\right]\left[\begin{array}{cc}
A_{1} & 0 \\
0 & A_{2}
\end{array}\right]\left[\begin{array}{l}
\mathcal{R}_{1} \\
\mathcal{R}_{2}
\end{array}\right]-\left[\begin{array}{ccc}
x & 0 & 0 \\
0 & y & 0 \\
0 & 0 & z
\end{array}\right]\left[\begin{array}{ccc}
\frac{d V_{1}}{d t} & 0 & 0 \\
0 & \frac{d V_{2}}{d t} & 0 \\
0 & 0 & \frac{d V_{3}}{d t}
\end{array}\right]\right) .
$$

In symbolic terms, the concentration-change ODEs are

$$
\frac{d \mathbf{s}}{d t}=\left(\operatorname{diag} C_{\mathbf{s}}\right)^{-1}\left(\mathbf{N v}-\operatorname{diag} S \cdot \operatorname{diag} \frac{d \mathbf{V}}{d t}\right)
$$

or, with explicit canonical concentration-change rate laws,

$$
\frac{d \mathbf{s}}{d t}=\left(\operatorname{diag} C_{\mathrm{s}}\right)^{-1}\left(\mathbf{N} \cdot \operatorname{diag} C_{\mathrm{r}} \cdot \mathcal{R}-\operatorname{diag} S \cdot \operatorname{diag} \frac{d \mathbf{V}}{d t}\right),
$$

with $\operatorname{diag} S$ a matrix of substance concentrations, and $\operatorname{diag}(d \mathbf{V} / d t)$ a diagonal matrix of rates at which the compartment volumes change.

\section{Moiety conservation}

Moiety-conserving cycles occur in reaction networks when different forms of a chemical moiety are interconverted while the sum of these forms remains constant [8]. Typical metabolic examples are the interconversions of ATP-ADP-AMP (the different forms of the adenylate moiety), NAD-NADH, and NADP-NADPH. Moiety conservation manifests in kinetics models as sets of dependent ODEs (see Appendix A). One of the ODEs in such a set must be replaced by a conservation equation.

As mentioned before, the amount-change ODEs for both Fig. 10A and B sum to zero, so that the sum of the mole amounts of the three substances are constant (conservation eqn. 60):

$$
n_{\mathrm{X}}+n_{\mathrm{Y}}+n_{\mathrm{Z}}=T_{\mathrm{N}}
$$


where $T_{\mathrm{N}}$ is the total mole amount of the three substances. If the rate equations are formulated in terms of concentrations instead of mole amounts the conservation equation can be expressed as

$$
V x+V y+V z=T_{\mathrm{N}}
$$

for Fig. 10A, while that for Fig. 10B is

$$
V_{1} x+V_{2} y+V_{3} z=T_{\mathrm{N}}
$$

In the single compartment system in Fig. 10A where the ODEs are linearly dependent the sum of concentrations is of course also constant:

$$
x+y+z=T_{\mathrm{C}}
$$

where $T_{C}$ is the total concentration of the three substances.

In models of systems in which compartmental volumes change with time, parameters such as the concentrations of intra-compartmental enzymes and total conserved moiety $T_{C}$ often need to remain constant because the synthesis and degradation of enzymes and conserved moiety is not part of the modelled reaction network. This implies that the amounts of enzymes and conserved moieties must change in proportion to the compartmental volume. Such constraints must be built into the rate equations by coupling the relevant parameters to a compartmental volume or to the entity that is used as an indicator of intra-compartmental volume, such as, for example, the biomass of a cell population.

\section{Advice to modellers and software authors}

Whether constructing a model of compartmentalised reaction networks or writing modelling software capable of handling compartmental models, one has to make strategic choices, especially if the models are to accommodate variable compartment sizes. How should one (or the software) construct the ODEs that describe the system dynamics? How much should happen under the hood of the modelling software and how much should be left for the modeller to specify?

First consider the situation where the model is to be defined from scratch using a computing platform such as Mathematica, Maple, Matlab/Octave or Maxima, i.e., where the ODEs, conservation equations and rate equations are all explicitly coded by the modeller. The analysis and presented in this paper clearly shows that the simplest and most widely applicable procedure by far is to cast the ODEs as rates of changes in the mole amounts of the network intermediate substances, and the amount-change rate equations as appropriately scaled canonical rate laws. Nothing of course prevents one from casting the model in terms of concentration-change ODEs, but why, when variable compartment sizes are in play, use

$$
\frac{d \mathbf{s}}{d t}=\left(\operatorname{diag} C_{\mathrm{s}}\right)^{-1}\left(\mathbf{N v}-\operatorname{diag} S \cdot \operatorname{diag} \frac{d \mathbf{V}}{d t}\right)
$$


in lieu of

$$
\frac{d \mathbf{n}}{d t}=\mathbf{N v} ?
$$

Besides relinquishing simplicity of formulation, one also incurs a computational overhead.

Dedicated modelling software for systems biology should preferably follow the same advice: use amount-change ODEs as default for compartmental models, let the software calculate the stoichiometry matrix $\mathbf{N}$ (or, when there is moiety conservation, $\mathbf{N}_{\mathbf{R}}$ and $\mathbf{L}$ ) from the stoichiometrically balanced reactions (see Appendix A), and leave specification of the rate laws from which $\mathbf{v}$ is calculated to the modeller. For fixed-size single compartment models, which are by far the most common, the obvious default is concentration-change ODEs and canonical concentration-change rate laws.

For me the simplest way of describing which aspects modelling software should take into consideration when defining compartmental models is to describe how our own modelling platform PySCeS [9] handles them.

By default PySCeS assumes that the model exists in a single, unit volume compartment. In this case it is unnecessary to define a compartment; the ODEs therefore describe changes in concentration per time; the rate equations are the canonical concentration-change rate equations. However, if a compartment is explicitly defined, PySCeS assumes that the ODEs describe changes in substance amount per time. Doing this affects how the model is defined in the input file. A compartment is defined as

Compartment: <name>, 〈size>, <dimensions>

where $\langle$ name $\rangle$ is the unique compartment name, and $\langle$ size $\rangle$ is the size of the compartment (i.e., length, volume or area) defined by the number of <dimensions $>(1,2$ or 3)

Compartment: Cell, 2.0, 3

Compartment: Memb, 1.5, 2

Two keywords are available for use with models that have one or more compartments defined. Both take a boolean (True/False) as their value:

- Species_In_Conc specifies whether the species symbols used in the rate equations represent a concentration (True, default) or an amount (False);

- Output_In_Conc tells PySCeS to output the results of numerical operations in concentrations (True, default) or in amounts (False), i.e., if Output_In_Conc: True PySCeS calculates the concentrations after each solver iteration by dividing mole amounts by the corresponding compartment sizes.

When compartments are defined, reactions and species are assigned a location using the $@$ symbol followed by the compartment name. The canonical rate laws must be scaled with the appropriate compartment size to form amount-change rate equations (SBML kinetic laws). In the following example Species_In_Conc:True, so that species symbols represent concentrations: 
R1@Cell:

$\mathrm{s} 1=\mathrm{s} 2$

$\operatorname{Cell} *(\mathrm{Vf} 1 / \mathrm{KS} 1) *(\mathrm{~s} 1-\mathrm{s} 2 / \mathrm{Keq} 1) /(1+\mathrm{s} 1 / \mathrm{KS} 1+\mathrm{s} 2 / \mathrm{KS} 2)$

Similarly, the compartment in which each species is located must be specified when the concentration is initialised:

$\mathrm{s} 10 \mathrm{Memb}=0.5$

$\mathrm{s} 2 @ \mathrm{Cell}=2.0 \mathrm{e}-4$

When Species_In_Conc:False, species symbols represent mole amounts and must be divided by the corresponding compartment size in the rate equations:

R1@Cell:

$$
\begin{aligned}
& \mathrm{s} 1=\mathrm{s} 2 \\
& \mathrm{Cell} *(\mathrm{Vf} 1 / \mathrm{KS} 1) *(\mathrm{~s} 1 / \mathrm{Memb}-(\mathrm{s} 2 / \mathrm{Cell}) / \mathrm{Keq} 1) / \\
& \quad(1+(\mathrm{s} 1 / \mathrm{Memb}) / \mathrm{KS} 1+(\mathrm{s} 2 / \mathrm{Cell}) / \mathrm{KS} 2)
\end{aligned}
$$

The units of all species (and anything that depends on them) are defined in terms of the Species_In_Conc keyword, and the user is responsible for making sure that the units of the initialised species are in line with the set value of Species_In_Conc. Here Species_In_Conc: False so that the species are initialised as mole amounts:

$$
\begin{aligned}
& \mathrm{s} 10 \text { Memb }=0.75 \\
& \mathrm{~s} 20 \mathrm{Cell}=4.0 \mathrm{e}-4
\end{aligned}
$$

When compartment sizes vary during the simulation there must be a way of specifying the additional ODEs that describe changes in compartment sizes per time. In PySCeS this is done via so-called rate rules, which are directly-encoded ODEs that are evaluated after the ODEs defined by the model stoichiometry and rate equations. The general form of a rate rule is

RateRule: <name> \{<formula $>$ \}

where <name> is the model attribute (e.g., compartment or parameter), the rate of change of which is described by the $\langle$ formula $\rangle$, for example (see Appendix D):

RateRule: Vcyt $\{(1.0 / \mathrm{Co}) *(\mathrm{R} 1()+(1-\mathrm{m} 1) * \mathrm{R} 2()+(1-\mathrm{m} 2) * \mathrm{R} 3()-\mathrm{R} 4()-\mathrm{R} 5())\}$

RateRule: Mem_Area $\{($ sigma_P $) * R 4()+($ sigma_L $) * R 5()\}$

\section{Acknowledgements}

This work would not have been possible without the many hours of intense discussion with my colleagues Jacky Snoep, Johann Rohwer, and Brett Olivier. Marnette Coetzee recast the MWC model into the form presented in the appendices to this paper. Ursula Kummer urged me to finally convert my notes into a paper so that she could quit having to say "Hofmeyr, personal communication". My heartfelt thanks to all of you. 


\section{References}

[1] Sauro, H. M. (2012). Enzyme Kinetics for Systems Biology. Ambrosius Publishing, 2nd edition.

[2] Klipp, E., Liebermeister, W., Wierling, C., and Kowald, A. (2016). Systems Biology: A Textbook. John Wiley \& Sons, 2nd edition.

[3] Atkins, P. and de Paula, J. (2006). Atkins' Physical Chemistry. Oxford University Press, Oxford, $8^{\text {th }}$ edition.

[4] Hucka, M., Bergmann, F. T., Chaouiya, C., Dräger, A., Hoops, S., Keating, S. M., König, M., Novère, N. L., Myers, C. J., Olivier, B. G., Sahle, S., Schaff, J. C., Sheriff, R., Smith, L. P., Waltemath, D., Wilkinson, D. J., and Zhang, F. (2019). The Systems Biology Markup Language (SBML): Language specification for Level 3 Version 2 Core (release 2).

[5] Le Novere, N., Bornstein, B., Broicher, A., Courtot, M., Donizelli, M., Dharuri, H., Li, L., Sauro, H., Schilstra, M., Shapiro, B., et al. (2006). Nucleic Acids Res. 34 (Suppl 1), D689-D691.

[6] Fisher, W. G., Yang, P.-C., Medikonduri, R. K., and Jafri, M. S. (2006). Annals of Biomedical Engineering 34, 1712-1728.

[7] van Eunen, K., Simons, S. M. J., Gerding, A., Bleeker, A., den Besten, G., Touw, C. M. L., Houten, S. M., Groen, B. K., Krab, K., Reijngoud, D.-J., and Bakker, B. M. (2013). PLoS Comput. Biol. 9, e1003186.

[8] Hofmeyr, J.-H. S., Kacser, H., and van der Merwe, K. J. (1986). Eur. J. Biochem. $155,631-641$.

[9] Olivier, B. G., Rohwer, J. M., and Hofmeyr, J.-H. S. (2005). Bioinformatics 21, 56056.

[10] Hofmeyr, J.-H. S. (2001). Metabolic control analysis in a nutshell. In Yi, T.-M., Hucka, M., Morohashi, M., and Kitano, H., editors, Proc. of the $2^{\text {nd }}$ International Conference on Systems Biology, pp. 291-300, Wisconsin. Omnipress.

[11] Strang, G. (1988). Linear algebra and its applications. Hartcourt Brace Jovanovich College Publishers, New York, $3^{\text {rd }}$ edition.

[12] Reder, C. (1988). J. Theor. Biol. 135, 175-201.

[13] Surovtsev, I. V., Morgan, J. J., and Lindahl, P. A. (2007). J. Theor. Biol. 244, 154-166. 


\section{A. The kinetic model}

In keeping with my recommendation that kinetic models of compartmentalised reaction networks are best cast in terms of mole amount changes instead of concentration changes, this description of the kinetic model has been adapted from [10] to describe changes in mole amounts instead of concentrations.

The kinetic model for any (metabolic) network of coupled chemical reactions and transport processes, whether compartmentalised or not, can be written as a set of nonlinear differential equations:

$$
\frac{d \mathbf{n}}{d t}=\mathbf{N v}[\mathbf{s}, \mathbf{p}]
$$

where, for a system of $n$ coupled reactions that inter-convert $m$ substances, $\mathbf{n}$ and $\mathbf{s}$ are $m$-dimensional column vectors of substance mole amounts and concentrations respectively, $\mathbf{N}$ is an $m \times n$-dimensional matrix of stoichiometric coefficients (the stoichiometry matrix), $\mathbf{v}$ is an $n$-dimensional column vector of reaction rates, and $\mathbf{p}$ is a $p$-dimensional column vector of parameters. Only variable substance mole amounts and concentrations are included in $\mathbf{n}$ and $\mathbf{s}$; substance concentrations which are buffered externally and can therefore be regarded as constant are considered to be included in the parameter vector $\mathbf{p}$.

In any systemic state the reaction rates $\mathbf{v}$ are functions of both substance concentrations $\mathbf{s}$ and parameters $\mathbf{p}$ such as kinetic constants and fixed external concentrations. That rates depend on substance concentrations is reflected in $\mathbf{v}[\mathbf{s}, \mathbf{p}]$ with the assumption that $\mathbf{s}$ is calculated from mole amounts and the appropriate compartment sizes after each iteration of the ODE solver.

The structure or topology of the reaction network is embodied in the stoichiometry matrix $\mathbf{N}$. An element $c_{i j}$ of $\mathbf{N}$ is the stoichiometric number, usually an integer, with which substance $S_{i}$ participates in reaction $j$ (if $S_{i}$ is a reactant, $c_{i j}<0$; if a product, $c_{i j}>0$; otherwise, $c_{i j}=0$ ). Two invariant properties can be extracted from $\mathbf{N}$, namely (i) the conservation relationships that arise when the differential equations are not all linearly independent, and (ii) the steady-state flux relationships. Here I discuss only the first.

By Gaussian elimination to row echelon form (see, e.g., [11]) we can determine whether the rows of $\mathbf{N}$ (and, therefore, the differential equations themselves) are linearly independent. If they are independent then $r=m$, where $r$ is the rank of $\mathbf{N}$ (the number of independent equations). If $r<m$ then there are $m-r$ dependencies among the differential equations. Eliminating $m-r$ dependent rows of $\mathbf{N}$ leaves a reduced stoichiometry matrix, $\mathbf{N}_{\mathbf{R}}$, with $r$ independent rows. $\mathbf{N}$ and $\mathbf{N}_{\mathbf{R}}$ can be related by constructing a link matrix $\mathbf{L}$ with dimensions $m \times r$ so that $\mathbf{N}=\mathbf{L N}_{\mathbf{R}}$ [12]. If $\mathbf{N}$ is re-arranged so that the independent rows come first, then $\mathbf{L}$ and the mole amount 
vector $\mathbf{n}$ that corresponds to the rows of the re-arranged $\mathbf{N}$ have the structure

$$
\mathbf{L}=\left[\begin{array}{c}
\mathbf{I}_{r} \\
\mathbf{L}_{0}
\end{array}\right] \quad \text { and } \quad \mathbf{n}=\left[\begin{array}{c}
\mathbf{n}_{\mathbf{i}} \\
\mathbf{n}_{\mathbf{d}}
\end{array}\right]
$$

where $\mathbf{I}_{r}$ is an $r$-dimensional identity matrix and $\mathbf{L}_{0}$ an $(m-r) \times r$-dimensional matrix that expresses the dependent time derivatives in terms of the independent time derivatives (see eqn. 102 below); $\mathbf{n}_{\mathbf{i}}$ refers to independent and $\mathbf{n}_{\mathbf{d}}$ to dependent mole amounts.

Using these relationships the kinetic model in eqn. 97 can be written as

$$
\frac{d \mathbf{n}}{d t}=\mathbf{L N}_{\mathbf{R}} \mathbf{v}\left[\mathbf{s}_{\mathbf{i}}, \mathbf{s}_{\mathbf{d}}, \mathbf{p}\right]
$$

where the functional relationship $\mathbf{v}=\mathbf{v}\left[\mathbf{s}_{\mathbf{i}}, \mathbf{s}_{\mathbf{d}}, \mathbf{p}\right]$ emphasises the fact that the dependencies among the differential equations allows the partitioning of $\mathbf{n}$ into $r$ independent mole amounts $\mathbf{n}_{\mathbf{i}}$ and $m-r$ dependent mole amounts $\mathbf{n}_{\mathbf{d}}$. Now the kinetic model can be expanded into

$$
\frac{d}{d t}\left[\begin{array}{c}
\mathbf{n}_{\mathbf{i}} \\
\mathbf{n}_{\mathbf{d}}
\end{array}\right]=\mathbf{L N}_{\mathbf{R}} \mathbf{v}\left[\mathbf{s}_{\mathbf{i}}, \mathbf{s}_{\mathbf{d}}, \mathbf{p}\right]=\left[\begin{array}{c}
\mathbf{I}_{r} \\
\mathbf{L}_{0}
\end{array}\right] \mathbf{N}_{\mathbf{R}} \mathbf{v}\left[\mathbf{s}_{\mathbf{i}}, \mathbf{s}_{\mathbf{d}}, \mathbf{p}\right]
$$

which can be split into two equations:

$$
\begin{aligned}
\frac{d \mathbf{n}_{\mathbf{i}}}{d t} & =\mathbf{N}_{\mathbf{R}} \mathbf{v}\left[\mathbf{s}_{\mathbf{i}}, \mathbf{s}_{\mathbf{d}}, \mathbf{p}\right] \\
\frac{d \mathbf{n}_{\mathbf{d}}}{d t} & =\mathbf{L}_{0} \mathbf{N}_{\mathbf{R}} \mathbf{V}\left[\mathbf{s}_{\mathbf{i}}, \mathbf{s}_{\mathbf{d}}, \mathbf{p}\right]=\mathbf{L}_{\mathbf{0}} \frac{d \mathbf{n}_{\mathbf{i}}}{d t}
\end{aligned}
$$

which, when combined, give

$$
\frac{d \mathbf{n}}{d t}=\mathbf{L} \frac{d \mathbf{n}_{\mathbf{i}}}{d t}
$$

It is clear that if $\mathbf{L}_{0}$ is known we need only consider the kinetics as expressed by eqn. 101, as eqn. 102 allows the expression of the linear dependencies between the rates of change of substance mole amounts:

$$
\frac{d}{d t}\left(\mathbf{n}_{\mathbf{d}}-\mathbf{L}_{0} \mathbf{n}_{\mathbf{i}}\right)=\mathbf{0}
$$

where $\mathbf{0}$ is a null vector (a vector of zeros). This implies that

$$
\mathbf{n}_{\mathbf{d}}=\mathbf{L}_{\mathbf{0}} \mathbf{n}_{\mathbf{i}}+\mathbf{T}
$$


where $\mathbf{T}$ is an $(m-r)$-dimensional vector of constant (conserved) sums of mole amounts. The full mole amount vector $\mathbf{n}$ can therefore be expressed as a function of $\mathbf{n}_{\mathbf{i}}$ and $\mathbf{T}$ :

$$
\mathbf{s}=\left[\begin{array}{c}
\mathbf{n}_{\mathbf{i}} \\
\mathbf{n}_{\mathbf{d}}
\end{array}\right]=\left[\begin{array}{c}
\mathbf{I}_{r} \\
\mathbf{L}_{0}
\end{array}\right] \mathbf{n}_{\mathbf{i}}+\left[\begin{array}{l}
\mathbf{0} \\
\mathbf{T}
\end{array}\right]=\mathbf{L n}_{\mathbf{i}}+\left[\begin{array}{c}
\mathbf{0} \\
\mathbf{T}
\end{array}\right]
$$

where 0 represents an $r$-dimensional subvector of zeros.

\section{B. Example: A compartmentalised Lotka-Volterra system}

The first example of a compartmental model, adapted from [4], is a Lotka-Volterra system with an added reversible transport step between the compartments called cytosol and nucleus (step $\mathrm{T}$ in the network diagram in Fig. 11). The transport step is regarded simply as a membrane-associated process that spans the two three-dimensional compartments. The time-course simulation of this model exhibits damped oscillations.

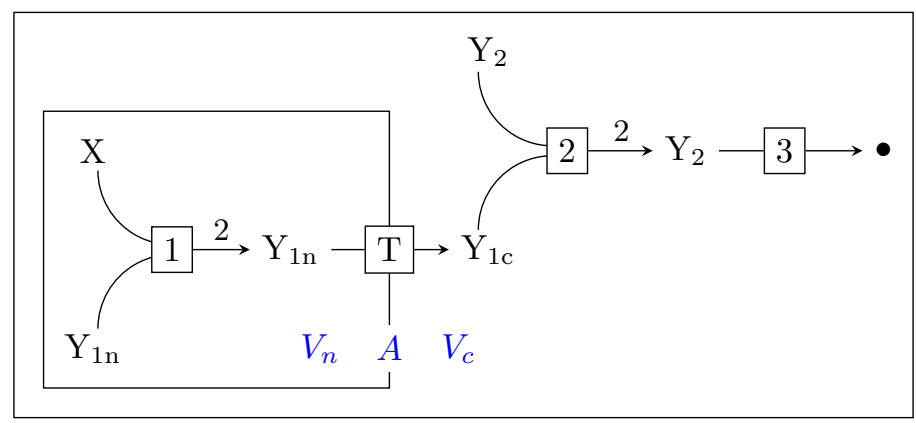

Fig. 11 Compartment example 7.8 on p. 130 of SBML Level 3 Version 2 Core Release 2 of 29 March 2019 [4]. The bullet represents a dummy species.

Assuming that the compartment volumes $V_{\mathrm{n}}, V_{\mathrm{c}}$ and the area, $A$, of the inner compartment membrane are known, the amount-change rate equations (SBML kinetic laws) are

$$
\begin{aligned}
& v_{1}=V_{\mathrm{n}} \cdot k_{1} \cdot x y_{1 \mathrm{n}} \\
& v_{\mathrm{T}}=A \cdot k_{\mathrm{T}} \cdot\left(y_{1 \mathrm{n}}-y_{1 \mathrm{c}}\right) \\
& v_{2}=V_{\mathrm{c}} \cdot k_{2} \cdot y_{1 \mathrm{c}} y_{2} \\
& v_{3}=V_{\mathrm{c}} \cdot k_{3} \cdot y_{2} .
\end{aligned}
$$

If $A$ is unknown, the amount-change rate equation for the transport reaction is

$$
v_{\mathrm{T}}=f_{\mathrm{T}} \cdot\left(y_{1 \mathrm{n}}-y_{1 \mathrm{c}}\right) \text {. }
$$


One could go even further and cast all the rate equations in terms of rate factors:

$$
\begin{aligned}
& v_{1}=f_{1} \cdot x y_{1 \mathrm{n}} \\
& v_{\mathrm{T}}=f_{\mathrm{T}} \cdot\left(y_{1 \mathrm{n}}-y_{1 \mathrm{c}}\right) \\
& v_{2}=f_{2} \cdot y_{1 \mathrm{c}} y_{2} \\
& v_{3}=f_{3} \cdot y_{2}
\end{aligned}
$$

Another possibility is that $A$ is unknown, but the mole amount $n_{\mathrm{T}}$ of transporters is known; in this case the amount-change rate equation for the transport reaction is

$$
v_{\mathrm{T}}=n_{\mathrm{T}} \cdot\left(y_{1 \mathrm{n}}-y_{1 \mathrm{c}}\right) \text {. }
$$

The amount-change ODEs are ( $\mathrm{X}$ is assumed to be fixed):

$$
\frac{d}{d t}\left[\begin{array}{l}
n_{Y_{1 \mathrm{n}}} \\
n_{\mathrm{Y}_{1 \mathrm{c}}} \\
n_{\mathrm{Y}_{2}}
\end{array}\right]=\left[\begin{array}{cccc}
1 & -1 & 0 & 0 \\
0 & 1 & -1 & 0 \\
0 & 0 & 1 & -1
\end{array}\right]\left[\begin{array}{c}
v_{1} \\
v_{\mathrm{T}} \\
v_{2} \\
v_{3}
\end{array}\right] .
$$

Appendix C provides the PySCeS input file that describes the Lotka-Volterra model, and a graph of the time-simulation showing damped oscillations.

\section{PySCeS model definition for the compartmentalised Lotka-Volterra system}

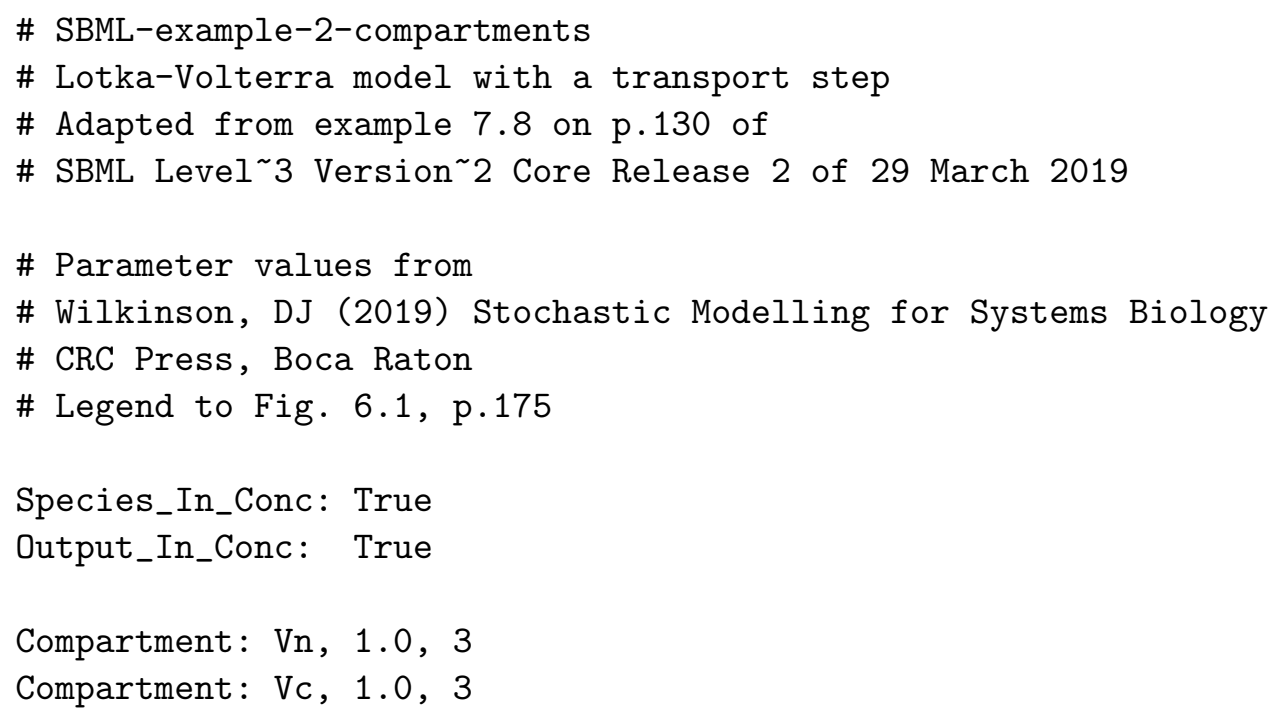


Compartment: A, 1.0, 2

FIX: X dummy

R1@V: $X+Y 1 n=\{2\} Y 1 n$

$\mathrm{Vn} * \mathrm{k} 1 * \mathrm{X} * \mathrm{Y} 1 \mathrm{n}$

$\mathrm{R} 2 @ \mathrm{~A}: \mathrm{Y} 1 \mathrm{n}=\mathrm{Y} 1 \mathrm{c}$

$\mathrm{A} * \mathrm{kT} *(\mathrm{Y} 1 \mathrm{n}-\mathrm{Y} 1 \mathrm{c})$

R3@Vc: $Y 1 c+Y 2=\{2\} Y 2$

$\mathrm{Vc} * \mathrm{k} 2 * \mathrm{Y} 1 \mathrm{c} * \mathrm{Y} 2$

R4@Vc: Y2 > dummy

$\mathrm{Vc} * \mathrm{k} 3 * \mathrm{Y} 2$

\# Initialise variable species

$\mathrm{Y} 1 \mathrm{n} \otimes \mathrm{Vn}=4.0$

$\mathrm{Y} 1 \mathrm{c} @ \mathrm{~V} c=0.0$

$\mathrm{Y} 2 \mathrm{QV} \mathrm{c}=10.0$

\# Initialise fixed species

$\mathrm{X@V} \quad=1.0$

dummy@V $=0.0$

\# Initialise parameters

$\mathrm{k} 1=1.0$

$\mathrm{k} 2=0.1$

$\mathrm{k} 3=0.1$

$\mathrm{kT}=5.0$

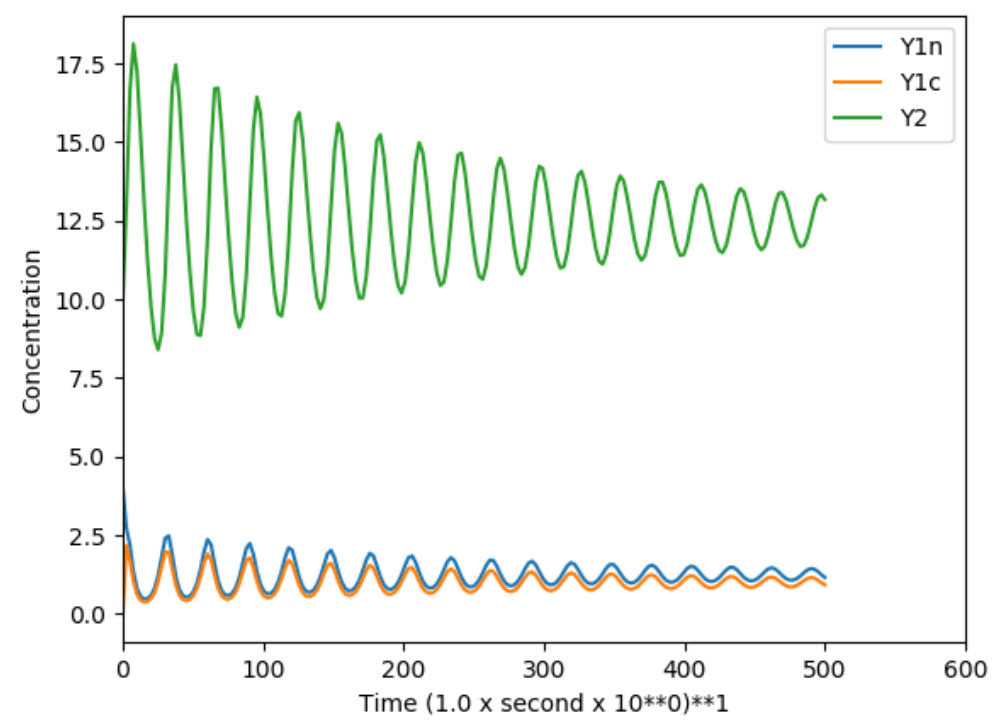




\section{Example: Minimal whole-cell model (MWC-model)}

The MWC-model was developed by the group of Paul Lindahl [13] and is an excellent example of a single compartment system the size of which changes. The reaction scheme in Fig. 12 shows a cytosolic compartment with volume $V_{\text {cyt }}$ encapsulated by a 2-dimensional membrane with area $A_{\mathrm{mem}}$. How the cytosolic volume and the membrane surface area changes as the cell grows is determined by (and in turn determines) the dynamics of a set of chemical reactions that occur in the cell.

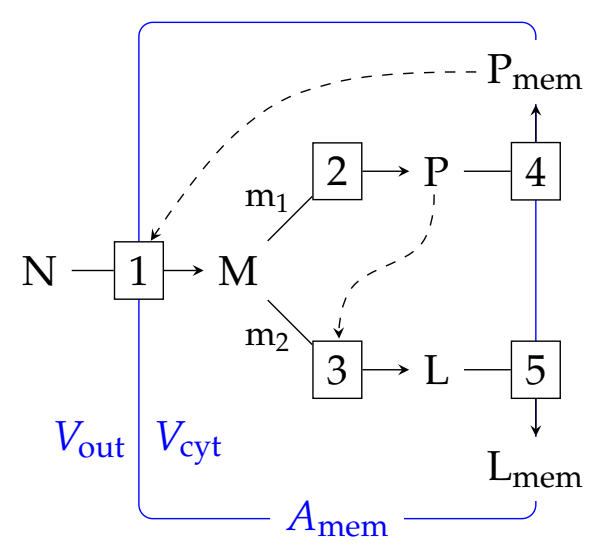

Fig. 12 Scheme of the minimal whole-cell model [13].

The cytosolic compartment contains three components: the metabolome + genome (M), the lipidome (L), and the proteome (P). The membrane consists exclusively of membrane proteins $\mathrm{P}_{\text {mem }}$ and phospholipids $\mathrm{L}_{\mathrm{mem}}$.

The five reactions are:

R1: $\mathrm{N} \longrightarrow \mathrm{M}$ (nutrients $\mathrm{N}$ are imported by a membrane-associated process into the cell and converted to $\mathrm{M}$; the process is catalysed by the membrane proteins $\mathrm{P}_{\mathrm{mem}}$ ). The extracellular concentration $n$ of $\mathrm{N}$ is assumed to be constant.

R2: $m_{1} \mathrm{M} \longrightarrow \mathrm{P}$ ( $m_{1}$ molecules of $\mathrm{M}$ form one $\mathrm{P}$ molecule $)$.

R3: $m_{2} \mathrm{M} \longrightarrow \mathrm{L}$ ( $m_{2}$ molecules of $\mathrm{M}$ form one $\mathrm{L}$ molecule; the process is catalysed by the proteome $\mathrm{P})$.

R4: $\mathrm{P} \longrightarrow \mathrm{P}_{\text {mem }}$ (a molecule of $\mathrm{P}$ is incorporated in the membrane in a membrane-associated process).

$\mathrm{R} 5: \mathrm{L} \longrightarrow \mathrm{L}_{\text {mem }}$ (a molecule of $\mathrm{L}$ is incorporated in the membrane in a membrane-associated process). 
The canonical concentration-change rate laws provided for the five reactions are:

$$
\begin{aligned}
& \mathcal{R}_{1}=k_{1} p_{\text {mem }} n \\
& \mathcal{R}_{2}=k_{2} m \\
& \mathcal{R}_{3}=k_{3} m p^{2} \\
& \mathcal{R}_{4}=k_{4} p \\
& \mathcal{R}_{5}=k_{5} l
\end{aligned}
$$

where $n, m, p, l$, and $p_{\text {mem }}$ are concentrations.

The dynamics of the MWC-model will now be described solely in terms of mole amounts, i.e., nowhere will concentrations appear as system variables. At the expense of expanding the concentration terms in the rate equations, the formulation of the model itself is the simplest, most transparent, and dare I say the most elegant, it can be.

The amount-change rates for the five reactions are:

$$
\begin{aligned}
& v_{1}=A_{\mathrm{mem}} \mathcal{R}_{1}=A_{\mathrm{mem}} \cdot k_{1}\left(\frac{n_{\mathrm{P}_{\mathrm{mem}}}}{A_{\mathrm{mem}}}\right)\left(\frac{n_{\mathrm{N}}}{V_{\mathrm{out}}}\right) \\
& v_{2}=V_{\mathrm{cyt}} \mathcal{R}_{2}=V_{\mathrm{cyt}} \cdot k_{2}\left(\frac{n_{\mathrm{M}}}{V_{\mathrm{cyt}}}\right) \\
& v_{3}=V_{\mathrm{cyt}} \mathcal{R}_{3}=V_{\mathrm{cyt}} \cdot k_{3}\left(\frac{n_{\mathrm{M}}}{V_{\mathrm{cyt}}}\right)\left(\frac{n_{\mathrm{P}}}{V_{\mathrm{cyt}}}\right)^{2} \\
& v_{4}=A_{\mathrm{mem}} \mathcal{R}_{4}=A_{\mathrm{mem}} \cdot k_{4}\left(\frac{n_{\mathrm{P}}}{V_{\mathrm{cyt}}}\right) \\
& v_{5}=A_{\mathrm{mem}} \mathcal{R}_{5}=A_{\mathrm{mem}} \cdot k_{5}\left(\frac{n_{\mathrm{L}}}{V_{\mathrm{cyt}}}\right)
\end{aligned}
$$

The amount-change ODEs for the substances are

$$
\begin{aligned}
\frac{d n_{\mathrm{M}}}{d t} & =v_{1}-m_{1} v_{2}-m_{2} v_{3} \\
\frac{d n_{\mathrm{P}}}{d t} & =v_{2}-v_{4} \\
\frac{d n_{\mathrm{L}}}{d t} & =v_{3}-v_{5} \\
\frac{d n_{\mathrm{P}_{\mathrm{mem}}}}{d t} & =v_{4} \\
\frac{d n_{\mathrm{L}_{\mathrm{mem}}}}{d t} & =v_{5} .
\end{aligned}
$$

How the cytosolic volume and the membrane area change in time as the cell grows rests on two assumptions:

- The osmotic pressure in the cytosolic volume, which is determined by the sum of the concentrations of $\mathrm{M}, \mathrm{P}$, and $\mathrm{L}$, must equal that of the external environment at all times. Using the van't Hoff equation $\pi=C R T$ (where $\pi$ is the osmotic pressure, $C$ the solute concentration, $R$ the universal gas constant, and $T$ the absolute temperature), the 
cytosolic volume of the cell can be calculated as

$$
V_{\text {cyt }}=\frac{R T}{\pi}\left(n_{\mathrm{M}}+n_{\mathrm{P}}+n_{\mathrm{L}}\right) .
$$

The osmotic pressure of an isotonic extracellular solution with concentration $C_{\text {out }}$ will be $\pi=C_{\text {out }} R T$. The cytosolic volume that will yield this osmotic pressure at specified mole amounts of $\mathrm{M}, \mathrm{P}$, and $\mathrm{L}$ is therefore

$$
V_{\text {cyt }}=\frac{1}{C_{\text {out }}}\left(n_{\mathrm{M}}+n_{\mathrm{P}}+n_{\mathrm{L}}\right) .
$$

The ODE for the change in cytosolic volume is

$$
\begin{aligned}
\frac{d V_{\mathrm{cyt}}}{d t} & =\frac{1}{C_{\text {out }}}\left(\frac{d n_{\mathrm{M}}}{d t}+\frac{d n_{\mathrm{P}}}{d t}+\frac{d n_{\mathrm{L}}}{d t}\right) \\
& =\frac{1}{C_{\mathrm{out}}}\left(v_{1}+\left(1-m_{1}\right) v_{2}+\left(1-m_{2}\right) v_{3}-v_{4}-v_{5}\right) .
\end{aligned}
$$

- The membrane surface is fully occupied by phospholipids $\mathrm{L}_{\mathrm{mem}}$ and membrane proteins $\mathrm{P}_{\text {mem }}$ (the authors refer to this as the area-filling-tiling assumption). The surface area can therefore be calculated from

$$
A_{\mathrm{mem}}=\sigma_{\mathrm{P}} n_{\mathrm{P}_{\mathrm{mem}}}+\sigma_{\mathrm{L}} n_{\mathrm{Lmem}}
$$

where $\sigma_{\mathrm{P}}$ and $\sigma_{\mathrm{L}}$ are the surface areas of one molecule of $\mathrm{P}$ and $\mathrm{L}$ respectively.

The ODE for the change in membrane surface area is therefore

$$
\frac{d A_{\mathrm{mem}}}{d t}=\sigma_{\mathrm{P}} \frac{n_{\mathrm{P}_{\mathrm{mem}}}}{d t}+\sigma_{\mathrm{L}} \frac{n_{\mathrm{L}_{\mathrm{mem}}}}{d t}=\sigma_{\mathrm{P}} v_{4}+\sigma_{\mathrm{L}} v_{5}
$$

These seven ODEs fully describe the dynamics of the system. If required, it is a trivial matter to define functions that monitor the time-dependent changes in derived variables such as the surface/volume ratio and substance concentrations. Appendix E provides the PySCeS input file that describes the MWC-model.

Contrast the above mole amount formulation of the model with one where everything is in terms of concentrations (the formulation that the authors used). The amount-change rate equations using concentrations are:

$$
\begin{array}{rlrl}
v_{1} & =A_{\mathrm{mem}} \cdot k_{1} p_{\mathrm{mem}} n & =A_{\mathrm{mem}} \mathcal{R}_{1} \\
v_{2} & =V_{\mathrm{cyt}} \cdot k_{2} m & =V_{\mathrm{cyt}} \mathcal{R}_{2} \\
v_{3} & =V_{\mathrm{cyt}} \cdot k_{3} m p^{2} & =V_{\mathrm{cyt}} \mathcal{R}_{3} \\
v_{4} & =A_{\mathrm{mem}} \cdot k_{4} p & & =A_{\mathrm{mem}} \mathcal{R}_{4} \\
v_{5} & =A_{\mathrm{mem}} \cdot k_{5} l & & =A_{\mathrm{mem}} \mathcal{R}_{5}
\end{array}
$$


and the ODEs for the substance concentrations, using the amount-change rate equations,

$$
\begin{aligned}
\frac{d m}{d t} & =\frac{1}{V_{\mathrm{cyt}}}\left(v_{1}-m_{1} v_{2}-m_{2} v_{3}\right)-\frac{m}{V_{\mathrm{cyt}}} \frac{d V_{\mathrm{cyt}}}{d t} \\
\frac{d p}{d t} & =\frac{1}{V_{\mathrm{cyt}}}\left(v_{2}-v_{4}\right)-\frac{p}{V_{\mathrm{cyt}}} \frac{d V_{\mathrm{cyt}}}{d t} \\
\frac{d l}{d t} & =\frac{1}{V_{\mathrm{cyt}}}\left(v_{3}-v_{5}\right)-\frac{l}{V_{\mathrm{cyt}}} \frac{d V_{\mathrm{cyt}}}{d t} \\
\frac{d p_{\mathrm{mem}}}{d t} & =\frac{1}{A_{\mathrm{mem}}} v_{4}-\frac{p_{\mathrm{mem}}}{A_{\mathrm{mem}}} \frac{d A_{\mathrm{mem}}}{d t} \\
\frac{d l_{\mathrm{mem}}}{d t} & =\frac{1}{A_{\mathrm{mem}}} v_{5}-\frac{l_{\mathrm{mem}}}{A_{\mathrm{mem}}} \frac{d A_{\mathrm{mem}}}{d t} .
\end{aligned}
$$

The compartment-size ODEs remain unchanged:

$$
\begin{aligned}
\frac{d V_{\mathrm{cyt}}}{d t} & =\frac{1}{C_{\mathrm{out}}}\left(v_{1}+\left(1-m_{1}\right) v_{2}+\left(1-m_{2}\right) v_{3}-v_{4}-v_{5}\right) \\
\frac{d A_{\mathrm{mem}}}{d t} & =\sigma_{\mathrm{P}} v_{4}+\sigma_{\mathrm{L}} v_{5}
\end{aligned}
$$

Using the canonical concentration-change rate equations the ODEs are

$$
\begin{aligned}
\frac{d m}{d t} & =\frac{A_{\mathrm{mem}}}{V_{\mathrm{cyt}}} \mathcal{R}_{1}-m_{1} \mathcal{R}_{2}-m_{2} \mathcal{R}_{3}-\frac{m}{V_{\mathrm{cyt}}} \frac{d V_{\mathrm{cyt}}}{d t} \\
\frac{d p}{d t} & =\mathcal{R}_{2}-\frac{A_{\mathrm{mem}}}{V_{\mathrm{cyt}}} \mathcal{R}_{4}-\frac{p}{V_{\mathrm{cyt}}} \frac{d V_{\mathrm{cyt}}}{d t} \\
\frac{d l}{d t} & =\mathcal{R}_{3}-\frac{A_{\mathrm{mem}}}{V_{\mathrm{cyt}}} \mathcal{R}_{5}-\frac{l}{V_{\mathrm{cyt}}} \frac{d V_{\mathrm{cyt}}}{d t} \\
\frac{d p_{\mathrm{mem}}}{d t} & =\mathcal{R}_{4}-\frac{p_{\mathrm{mem}}}{A_{\mathrm{mem}}} \frac{d A_{\mathrm{mem}}}{d t} \\
\frac{d l_{\mathrm{mem}}}{d t} & =\mathcal{R}_{5}-\frac{l_{\mathrm{mem}}}{A_{\mathrm{mem}}} \frac{d A_{\mathrm{mem}}}{d t}
\end{aligned}
$$

and

$$
\begin{aligned}
\frac{d V_{\text {cyt }}}{d t} & =\frac{1}{C_{\text {out }}}\left(A_{\text {mem }} \mathcal{R}_{1}+\left(1-m_{1}\right) V_{\text {cyt }} \mathcal{R}_{2}+\left(1-m_{2}\right) V_{\text {cyt }} \mathcal{R}_{3}-A_{\text {mem }} \mathcal{R}_{4}-A_{\text {mem }} \mathcal{R}_{5}\right) \\
\frac{d A_{\text {mem }}}{d t} & =\sigma_{\mathrm{P}} A_{\text {mem }} \mathcal{R}_{4}+\sigma_{\mathrm{L}} A_{\text {mem }} \mathcal{R}_{5} .
\end{aligned}
$$

Appendix E provides the PySCeS input file that describes the minimal whole-cell model, and graphs of how the species concentrations and the surface-to-volume ratio change with time.

\section{E. PySCeS model definition for the minimal whole-cell model}

Modelname: MWC-model 
Description: Minimal whole-cell model

\# Ivan V. Surovtsev, Jeffrey J. Morgan and Paul A. Lindahl

\# J. theor. Biol.244, 154-166, 2007

\# All species and ODE output in amounts

Species_In_Conc: False

Output_In_Conc: False

\# Compartment definition (name, initial value, dimensions)

Compartment: Vcyt, $1.0,3$

Compartment: Vout, 1.0, 3

Compartment: Mem_Area, 5.15898, 2

FIX : N

R1@Mem_Area: $\mathrm{N}=\mathrm{M}$

Mem_Area*k1*(Pmem/Mem_Area)*(N/Vout)

R2@Vcyt: $\{244\} \mathrm{M}=\mathrm{P} \# \mathrm{~m} 1$

Vcyt $* \mathrm{k} 2 *(\mathrm{M} / \mathrm{Vcyt})$

R3@Vcyt: $\{42\} \mathrm{M}=\mathrm{L} \# \mathrm{~m} 2$

Vcyt $* \mathrm{k} 3 *(\mathrm{M} / \mathrm{Vcyt}) *(\mathrm{P} / \mathrm{Vcyt}) * * 2$

R4@Mem_Area: $\mathrm{P}=$ Pmem

Mem_Area*k4*(P/Vcyt)

R5@Mem_Area: L $=$ Lmem

Mem_Area*k5*(L/Vcyt)

\# Rate rule definition

RateRule: Vcyt $\{(1.0 / \mathrm{Co}) *(\mathrm{R} 1()+(1-\mathrm{m} 1) * \mathrm{R} 2()+(1-\mathrm{m} 2) * \mathrm{R} 3()-\mathrm{R} 4()-\mathrm{R} 5())\}$

RateRule: Mem_Area $\{($ sigma_P $) * R 4()+($ sigma_L $) * R 5()\}$

\# Species initialisations

N@Vout $\quad=3.07$ e5

Pmem@Mem_Area $=192.86408217$

Lmem@Mem_Area $=41871.0515$

M@Vcyt $\quad=199693.0$

$\mathrm{L} @$ V cyt $\quad=102004$

P@Vcyt $=5303$

\# Parameter initialisations

$\mathrm{k} 1=0.89709 \mathrm{e}-3$

$\mathrm{k} 2=0.182027 \mathrm{e}-3$

$\mathrm{k} 3=0.17539 \mathrm{e}-9$ 


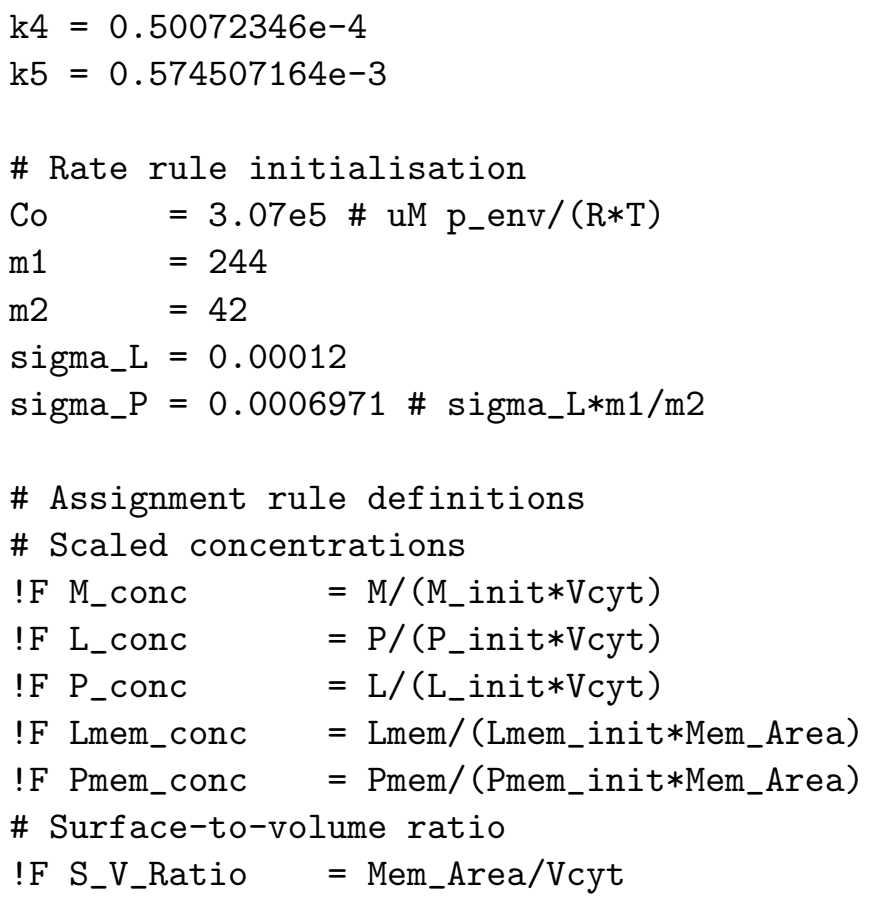

Simulate this model to time $=200$ and watch the scaled concentrations

M_conc, L_conc, P_conc, Lmem_conc, Pmem_conc and the surface/volume ratio $\mathrm{S}_{-}$V_Ratio.

" " "
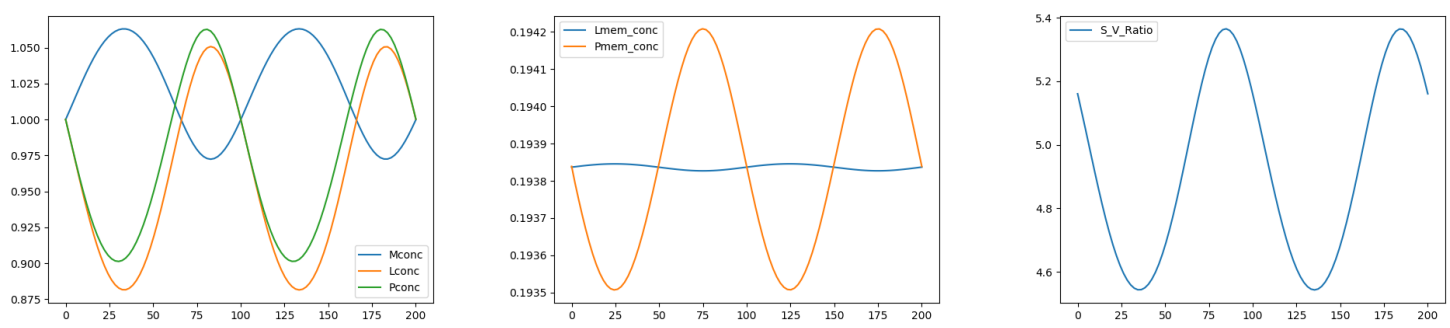